\title{
How an Eight-Membered Ring Alters the Rhodamine Chromophore
}

\author{
Yevgen M. Poronik, ${ }^{\dagger}$ Filip Ambicki, ${ }^{\dagger}$ Sheng-Ming Tseng, ${ }^{\S}$ Pi-Tai Chou, ${ }^{*, \S}$ Irena Deperasińska, ${ }^{*}$, Daniel T. \\ Gryko*,+ \\ ${ }^{\dagger}$ Institute of Organic Chemistry, Polish Academy of Sciences, Kasprzaka 44/52, 01-224 Warsaw, Poland \\ ${ }^{\ddagger}$ Institute of Physics Polish Academy of Sciences, Al. Lotników 32/46, 02-668 Warsaw, Poland \\ ${ }^{\S}$ Department of Chemistry, National Taiwan University, 1 Roosevelt Road Section 4, Taipei 106, Taiwan \\ E-mail: dtgryko@icho.edu.pl deper@ifpan.edu.pl chop@ntu.edu.tw
}

Table of Contents

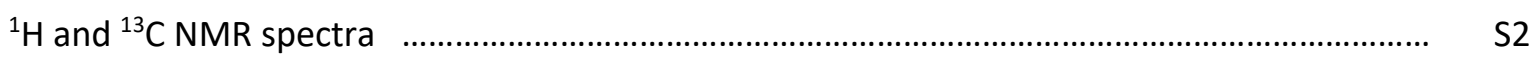

X-ray data structures for compounds 4b and 4c (Figures S1, S2, S3 and S4) .......................... S20

Calculations $\quad$ S23

Table S1. Comparison of the optimization results for 8U-Rh (4b), RB and MG ........................ S23

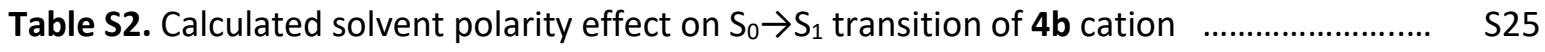

Figure S5. The ordering of molecular orbitals of $\mathbf{4 b}$ ion pair in solvents of different polarity $\quad$ S25

Table S3. Energies of $\mathrm{S}_{0} \rightarrow \mathrm{S}_{i}$ for ion pair $\mathrm{R}^{+} \mathrm{Cl}^{-}$of $\mathbf{4 b}$ in solvents of different polarity $\quad \ldots \ldots \ldots \ldots . . . . . . . \quad \mathrm{S} 26$

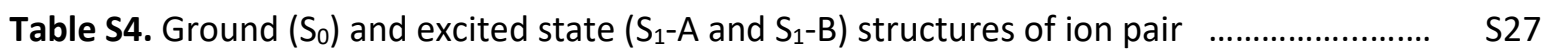

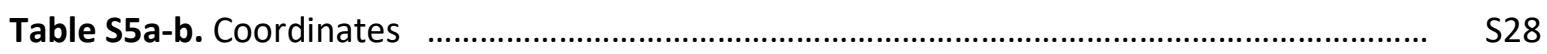

Mini Model $\quad$ S32

Table S6. HOMO and LUMO orbitals of $\mathrm{R}^{+}$optimized in $\mathrm{S}_{0}$ and $\mathrm{S}_{1}$ electronic state $\quad . . . . . . . . . . . . . . \quad \mathrm{S} 32$

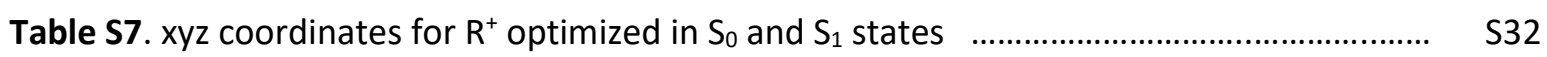

Table S8. The forms of $\mathrm{R}^{+} \mathrm{Cl}^{-}$optimized in ground and excited states ........................................ S33

Table S9. $x y z$ coordinates of $\mathrm{R}^{+} \mathrm{Cl}^{-}$forms of Mini Model .......................................................... S34

Table S10. The torsion angles for central ring of ground and excited state forms of $\mathrm{R}^{+} \mathrm{Cl}^{-} \quad \ldots \ldots . \quad \mathrm{S} 35$

Table S11. The vibration frequencies corresponding to the optimized forms of $\mathrm{R}^{+}$and $\mathrm{R}^{+} \mathrm{Cl}^{-} \quad \mathrm{S} 35$

$\begin{array}{ll}\text { Photoluminescence data } & \text { S38 }\end{array}$

Table S12. The photoluminescence quantum yields in polycrystalline state and in the $\begin{array}{ll}\text { ground powder. } & \text { S38 }\end{array}$

$\begin{array}{ll}\text { References } & \text { S38 }\end{array}$ 
$\mid$
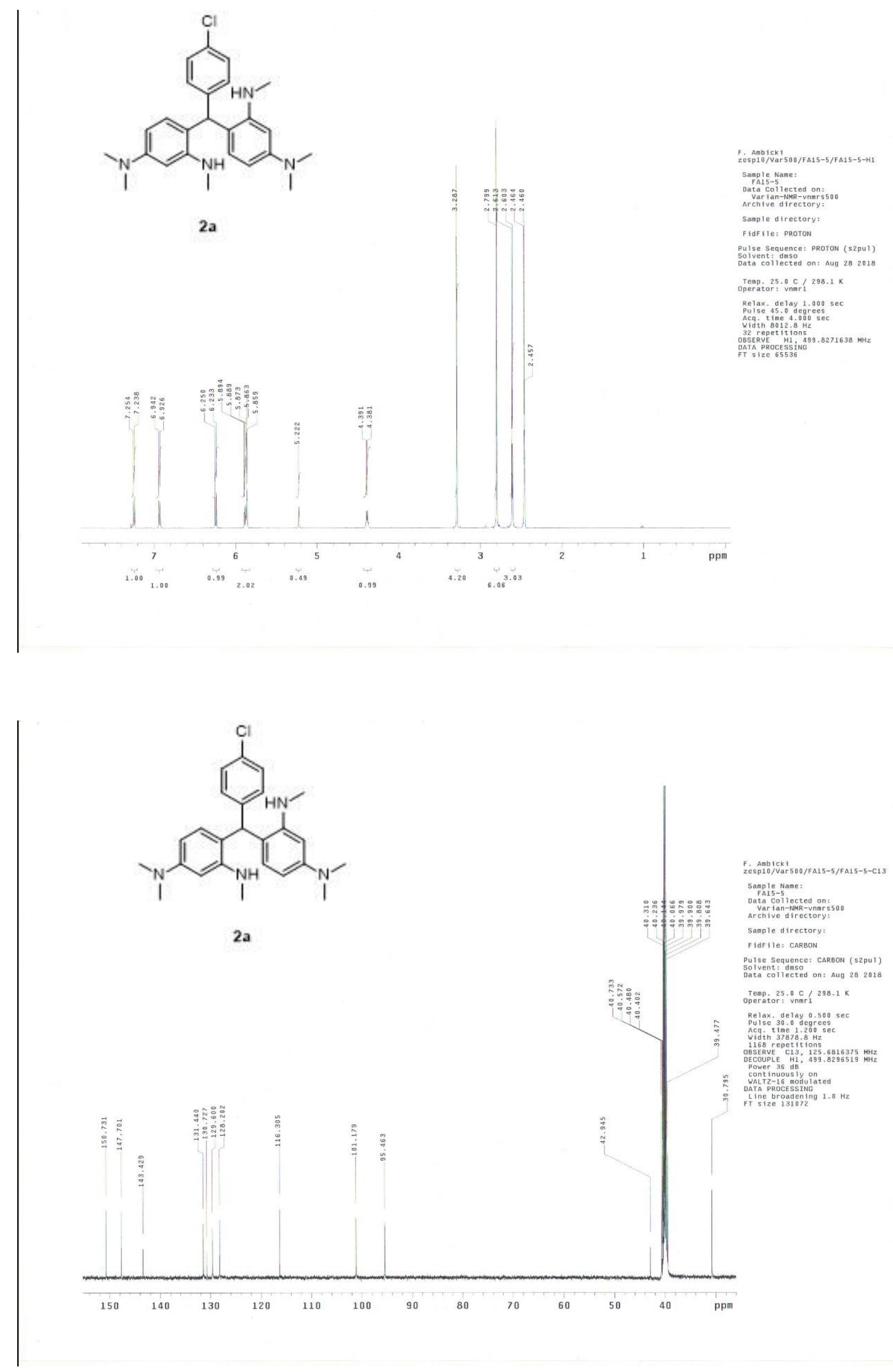

S2 


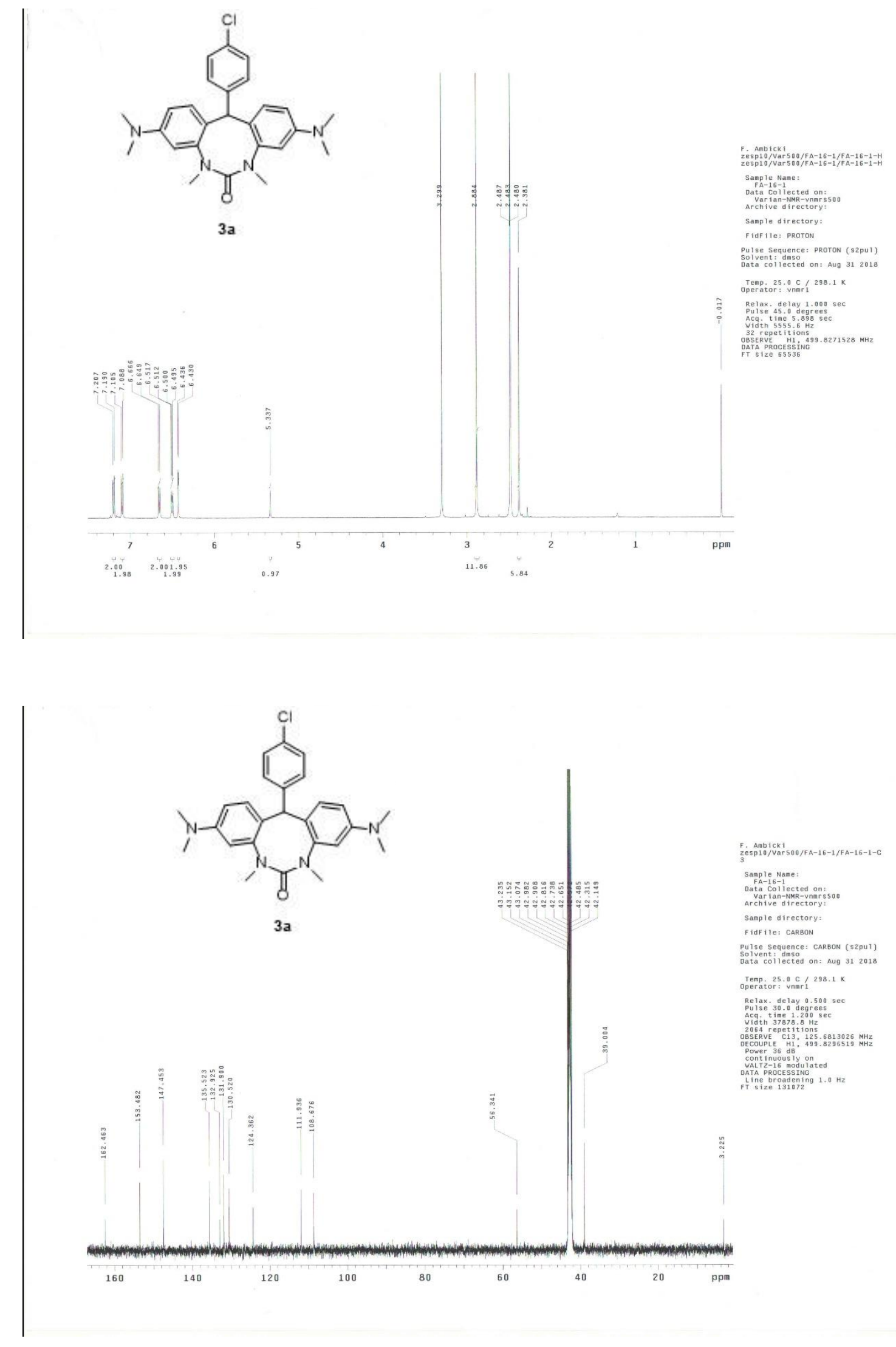




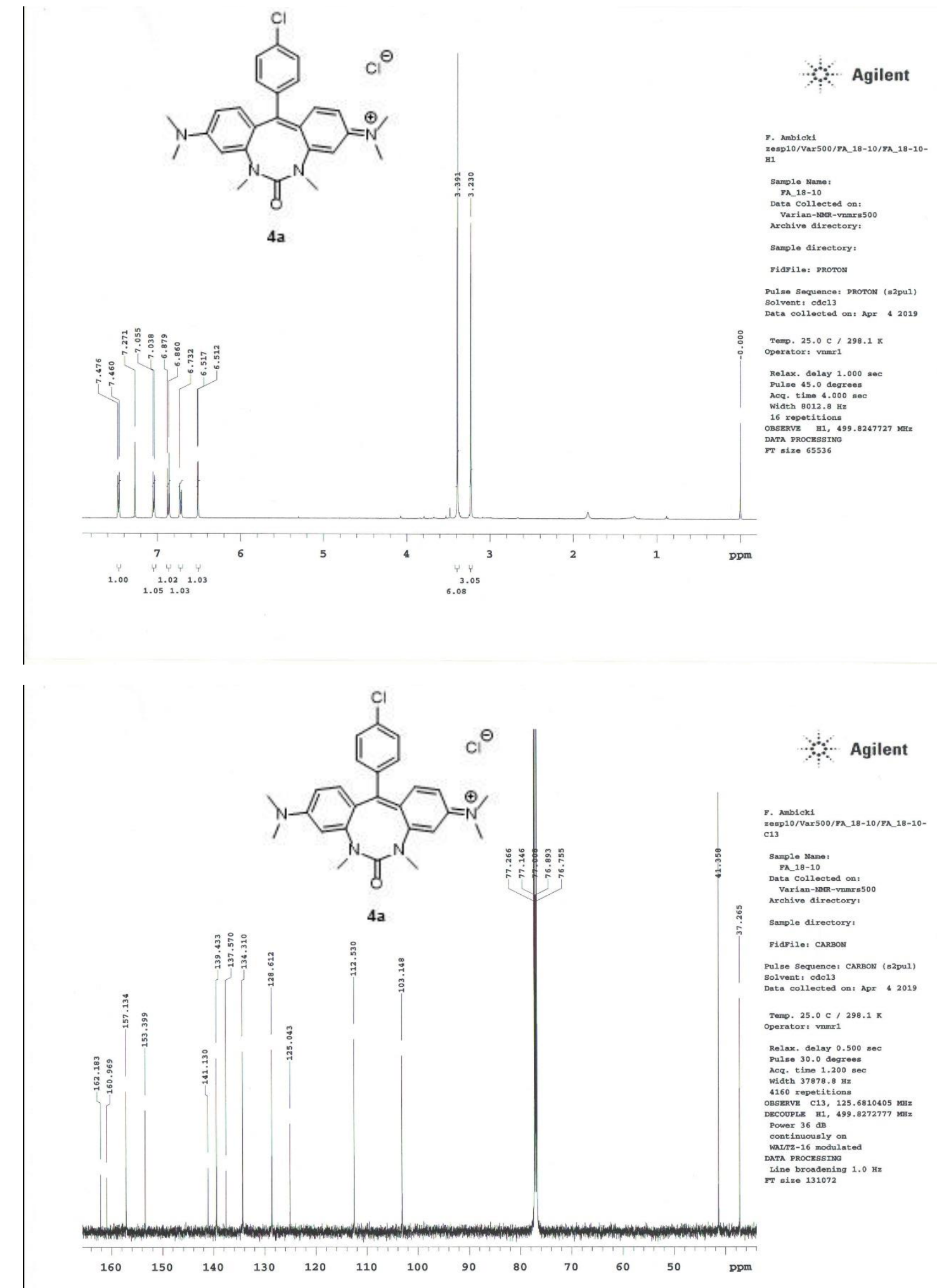



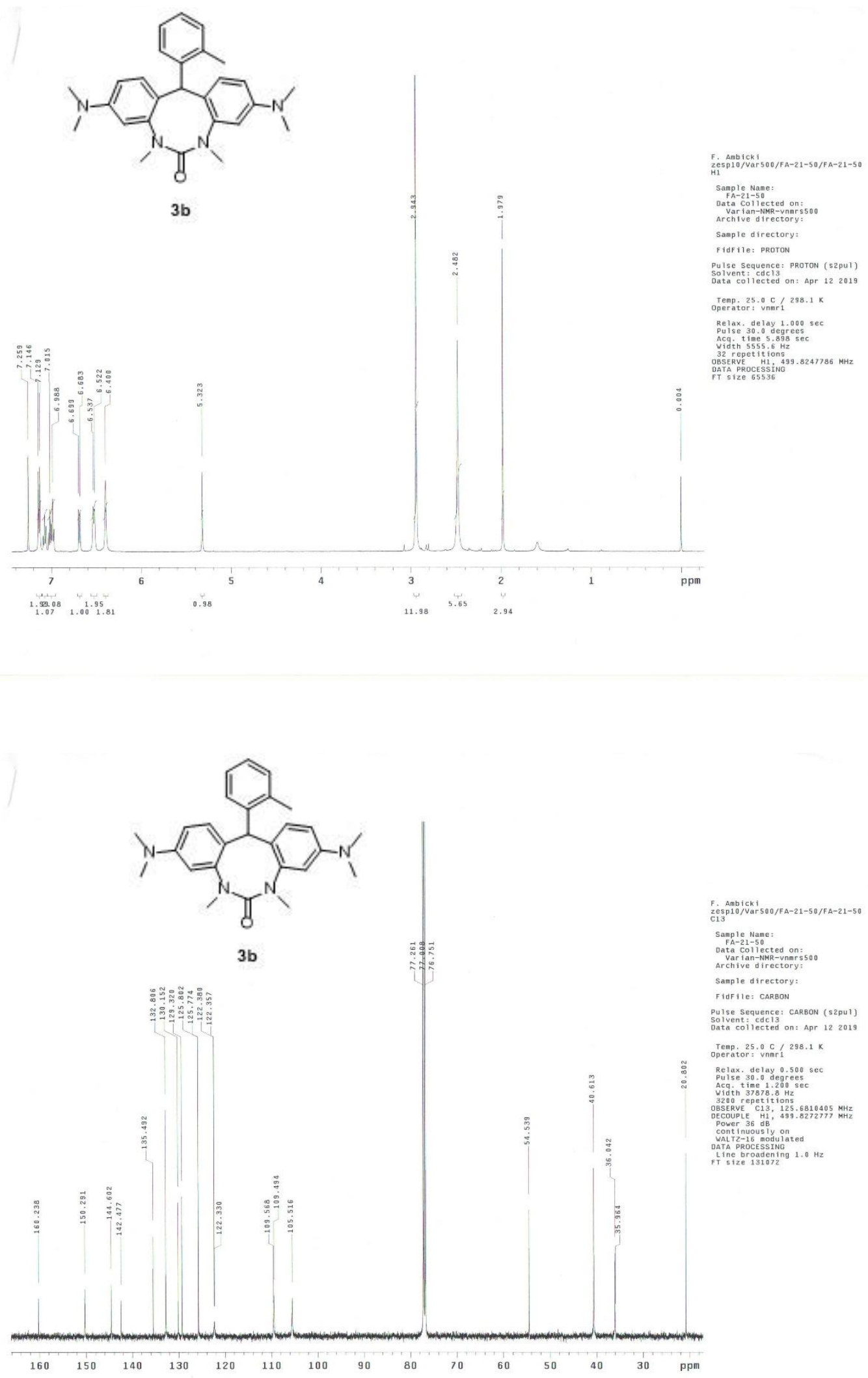

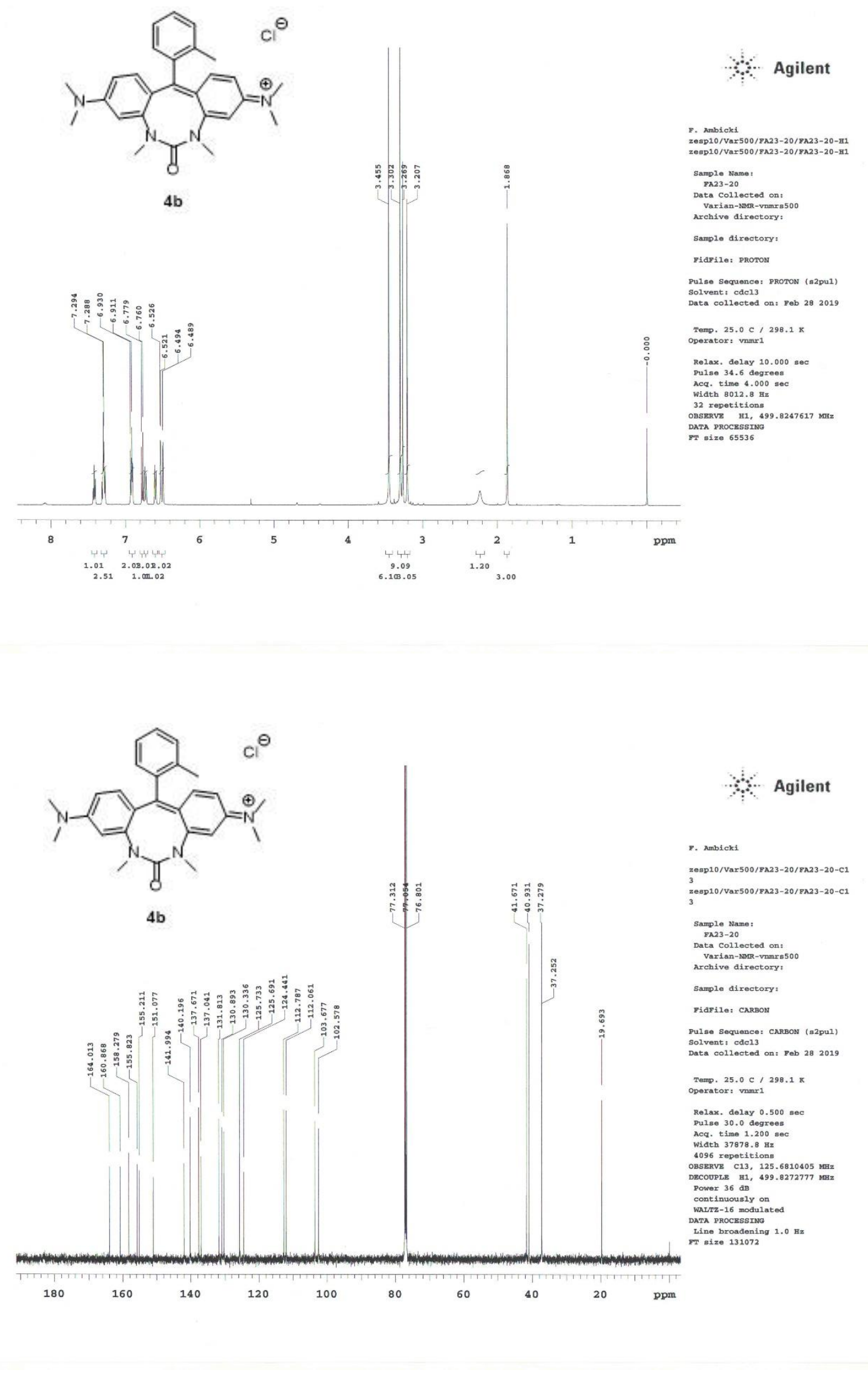

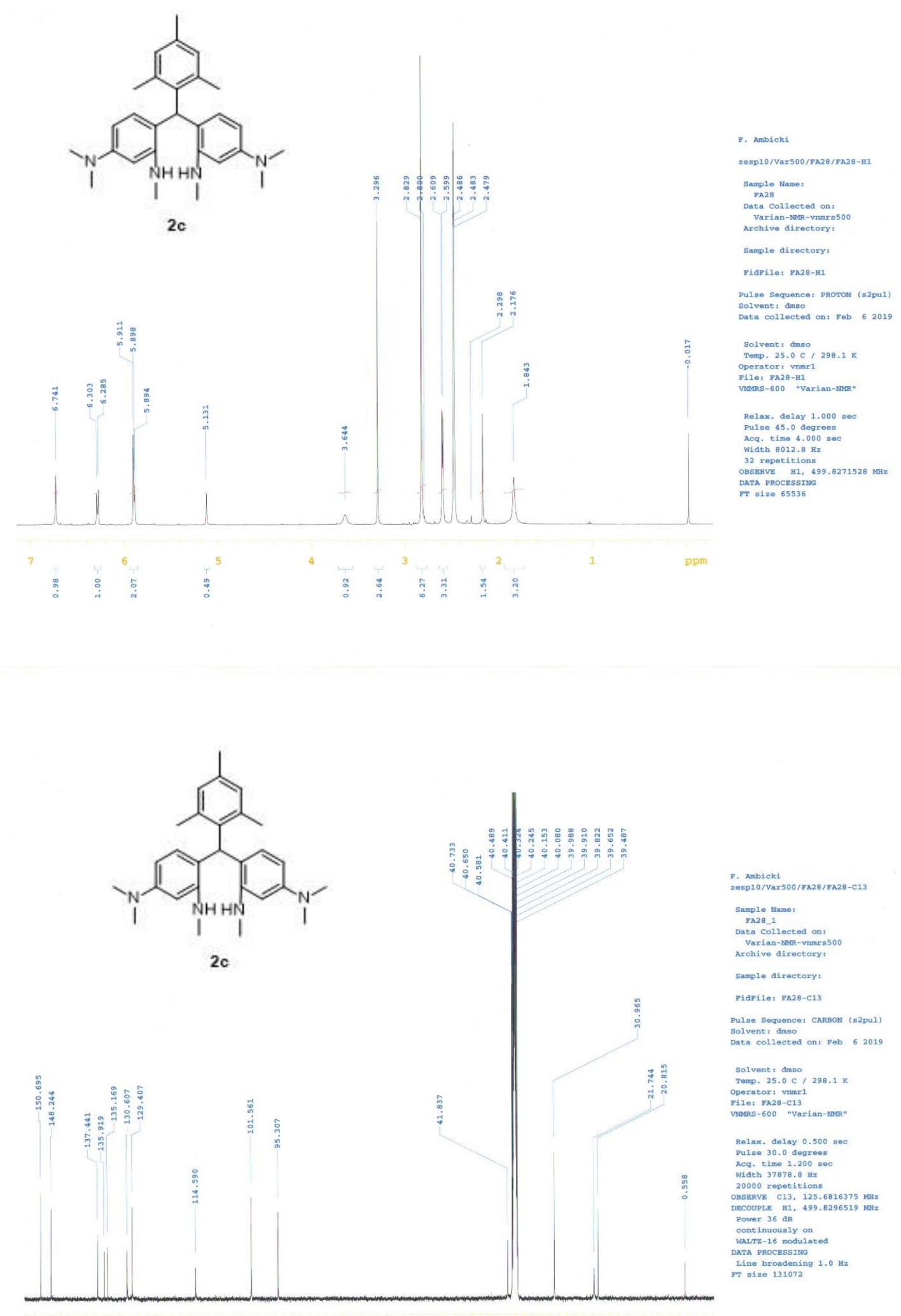

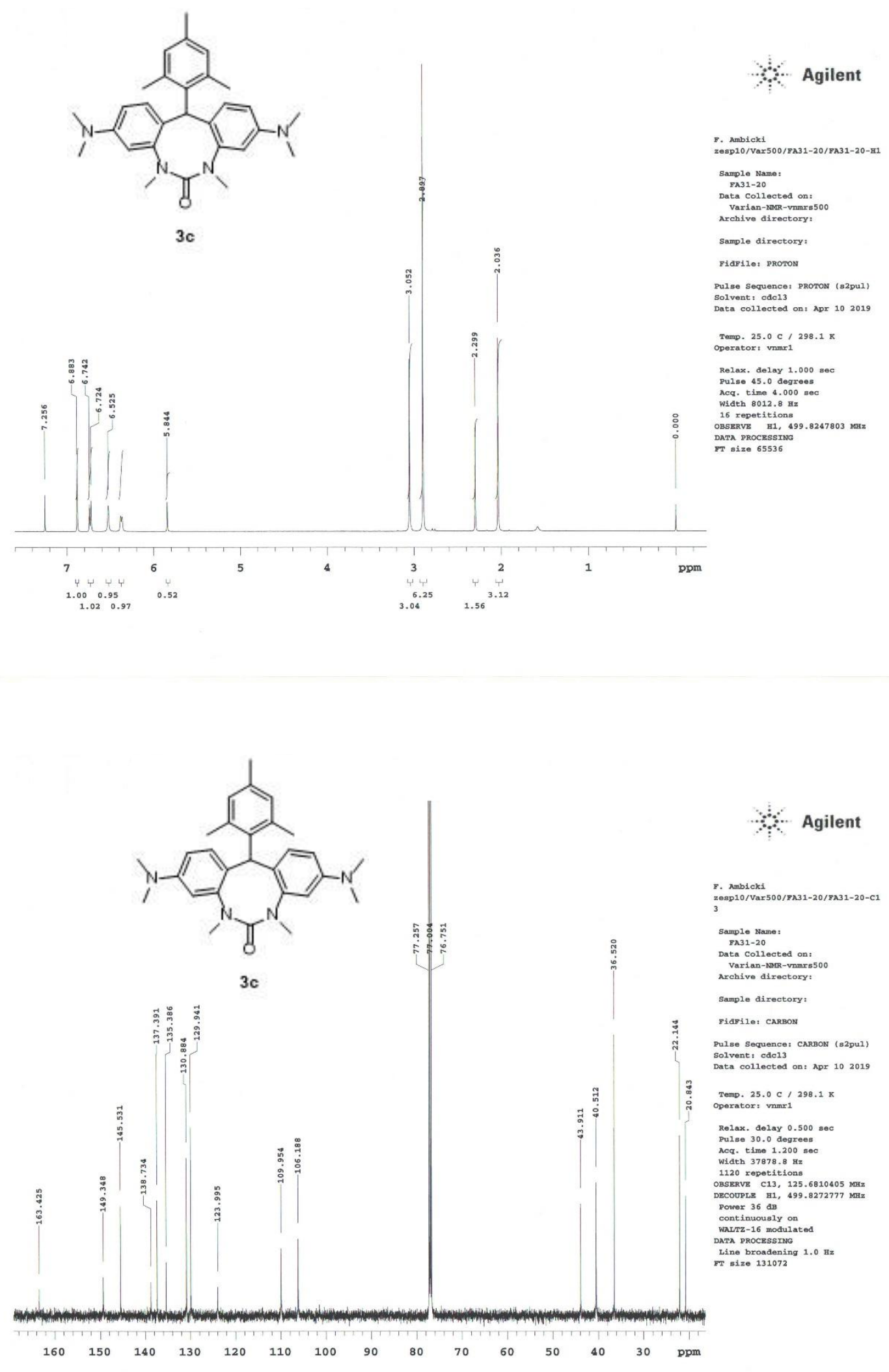

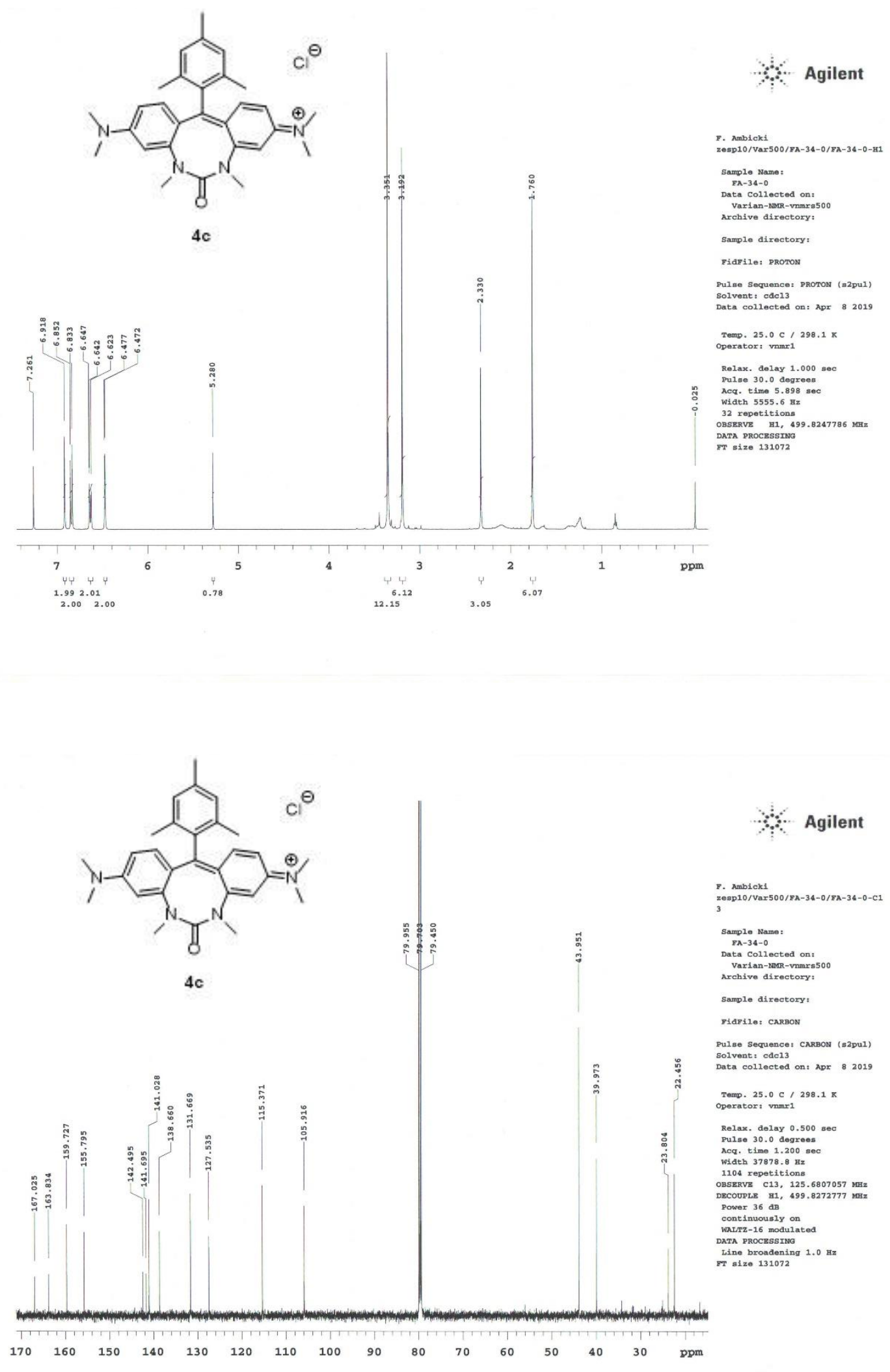

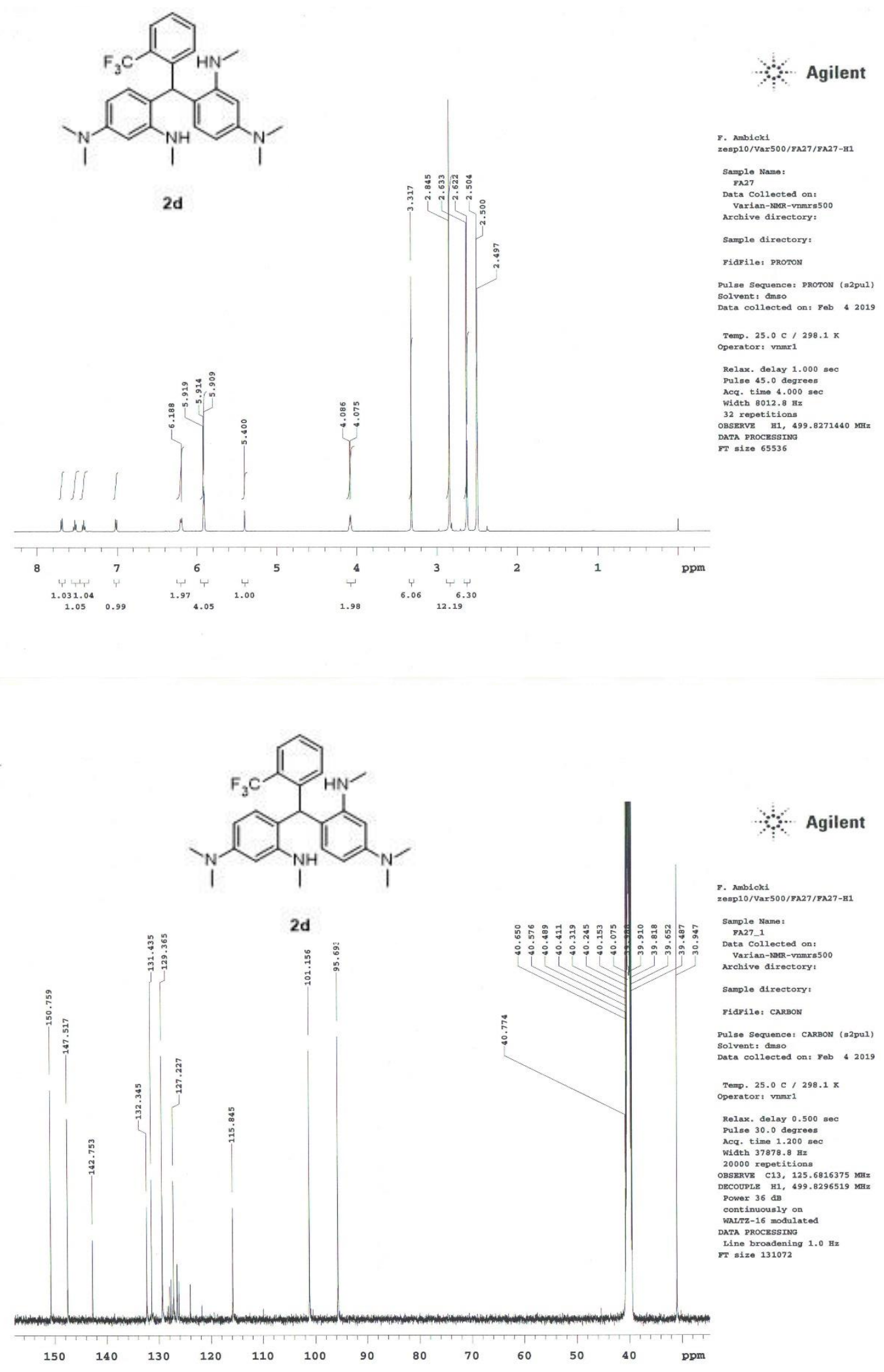
$\mid$

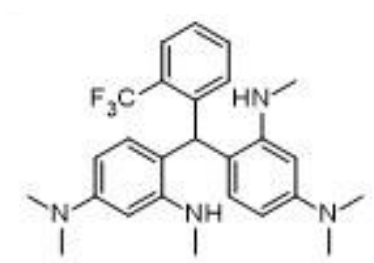

$2 d$

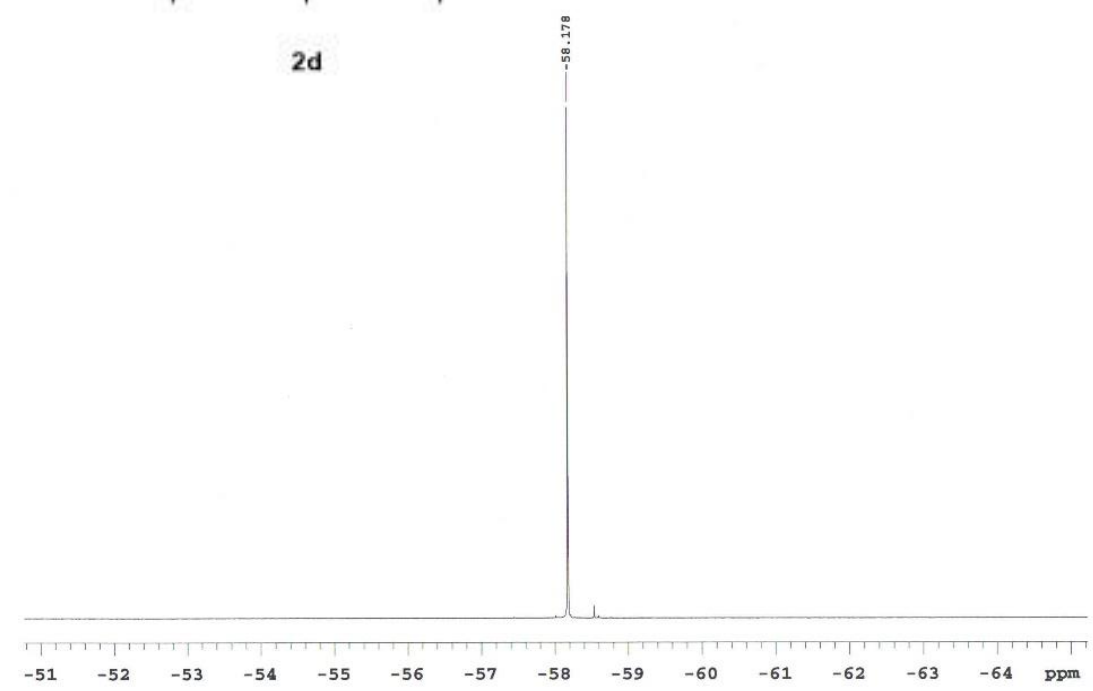

Agilent

F. Ambicki
zesp10/Var500/FA27/8R27-F19

Samp10 Name:
FA27

Data collected on:
varian-NMR-vmmreso

Archive directory:

Sample directory:

Piafile: rLooring

Pulae Sequence: FLVortars (s2pul)

Data collected on: Feb 42019

Temp. $25.0 \mathrm{C} / 298.1 \mathrm{~K}$
Operator: vnmr1

Relax. delay $1.000 \mathrm{sec}$

Pulse 30.0 degrees
Acq. time $0.839 \mathrm{sec}$

wiath $39062.5 \mathrm{~Hz}$

128 repotitions

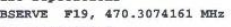

DATA ProCgsSIN
gize 65536 

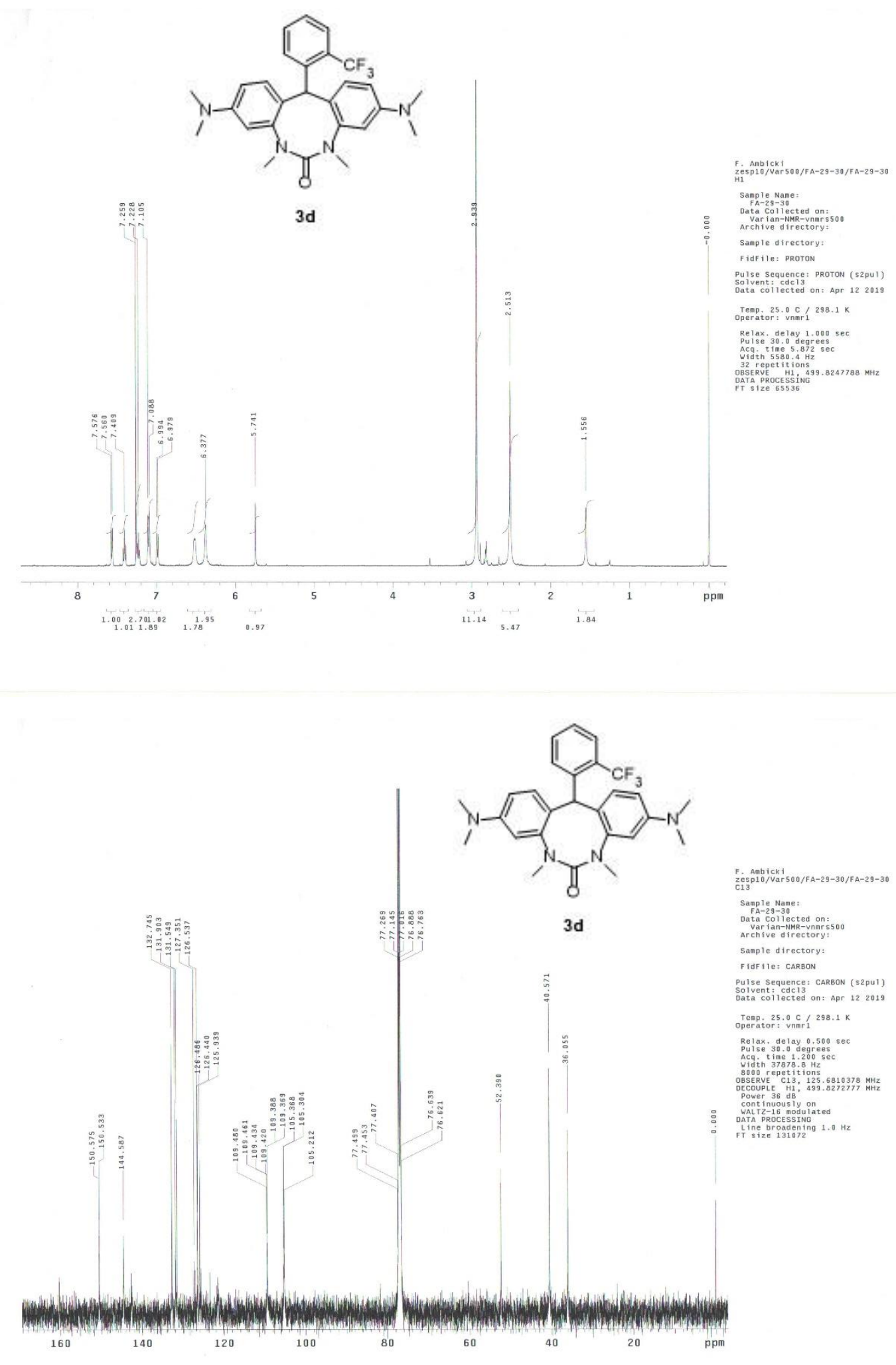


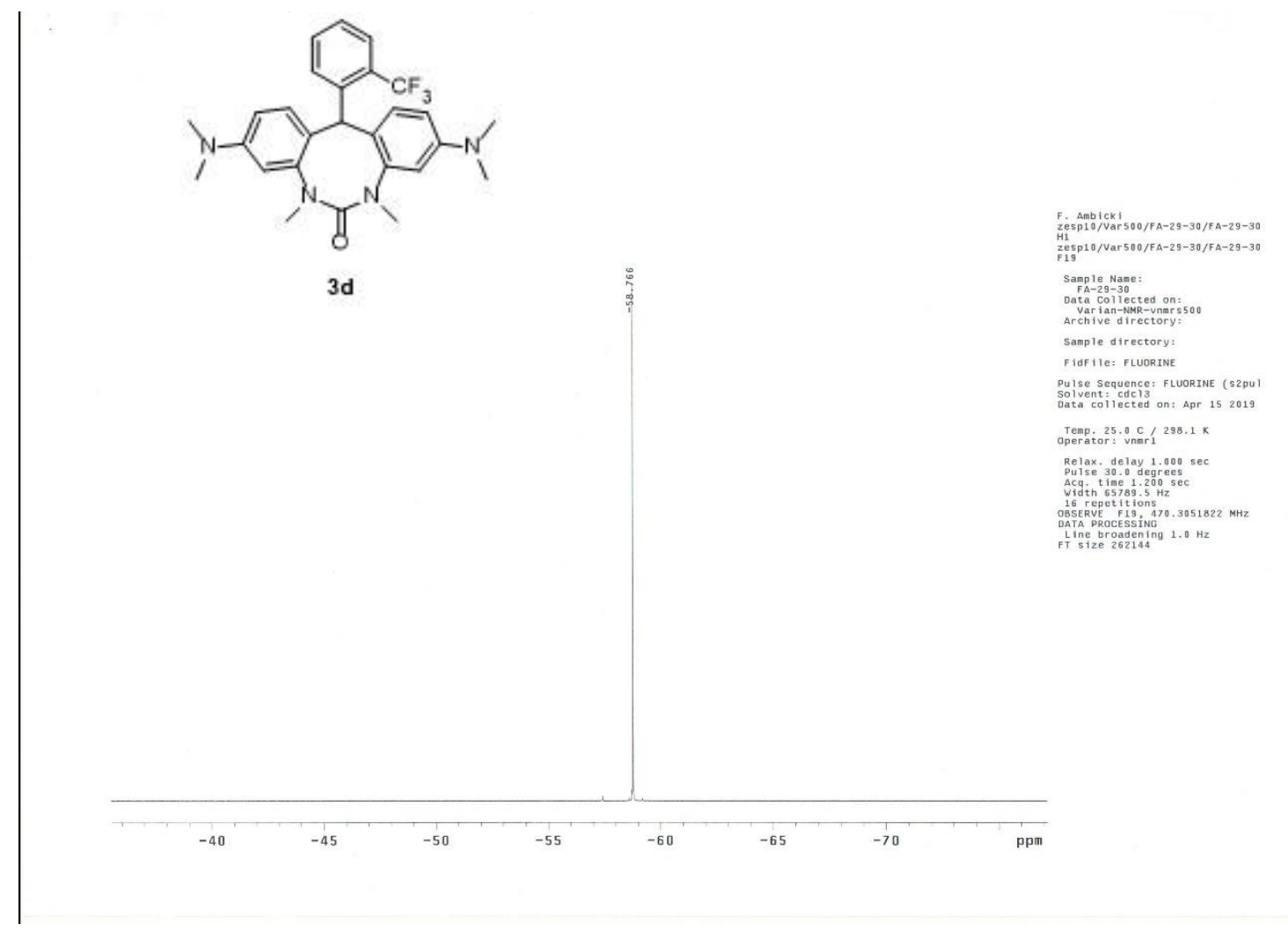



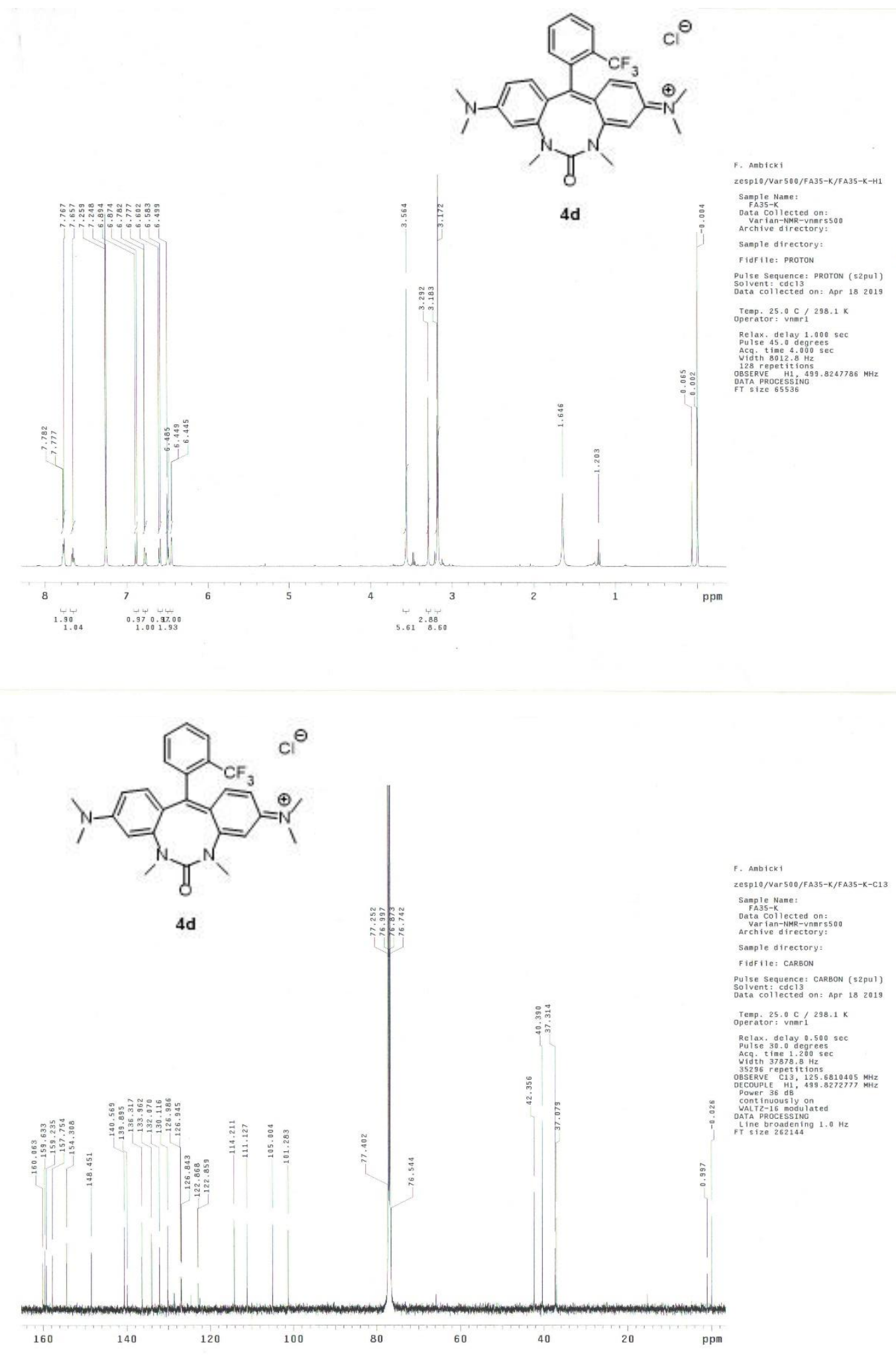


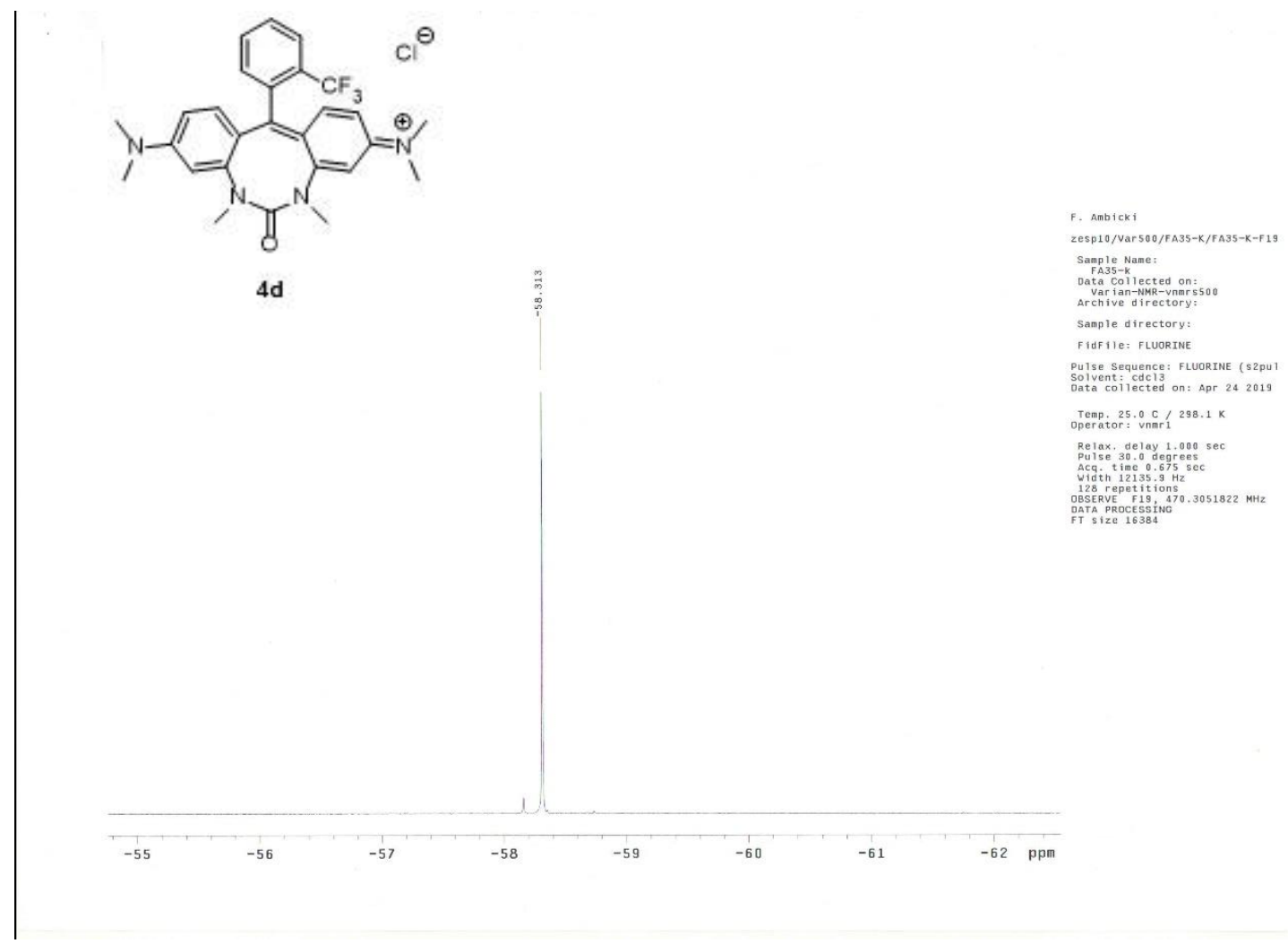




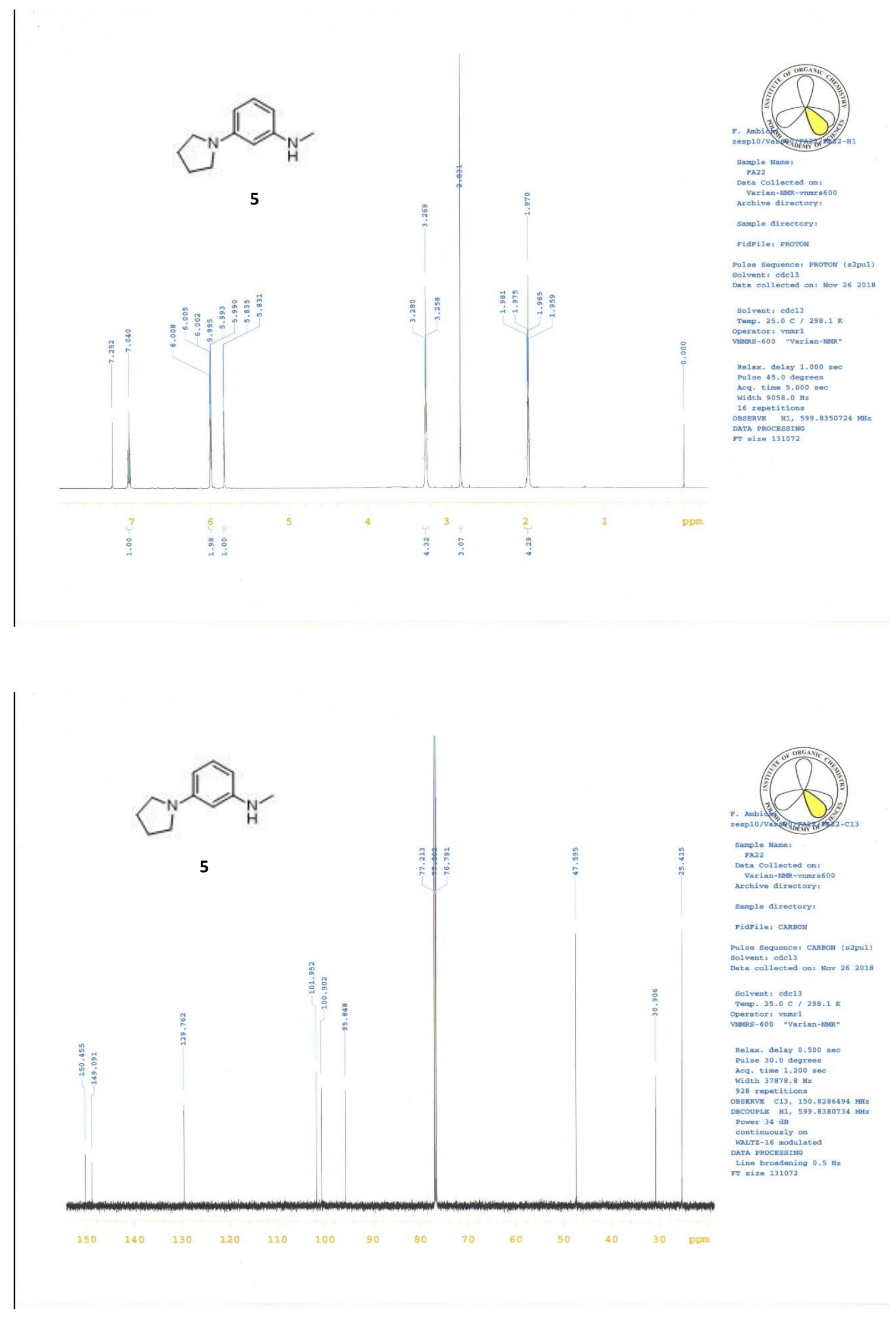



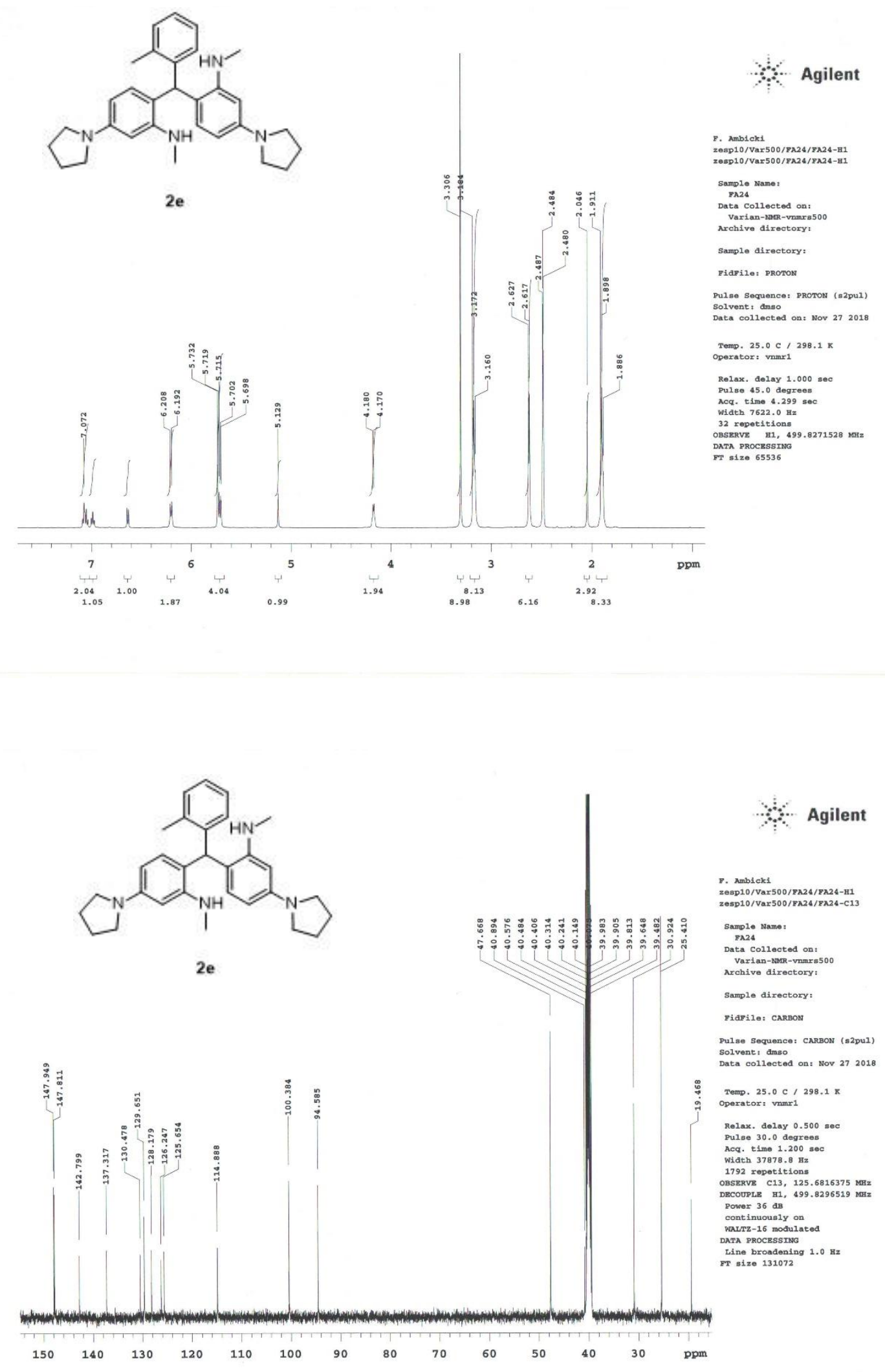

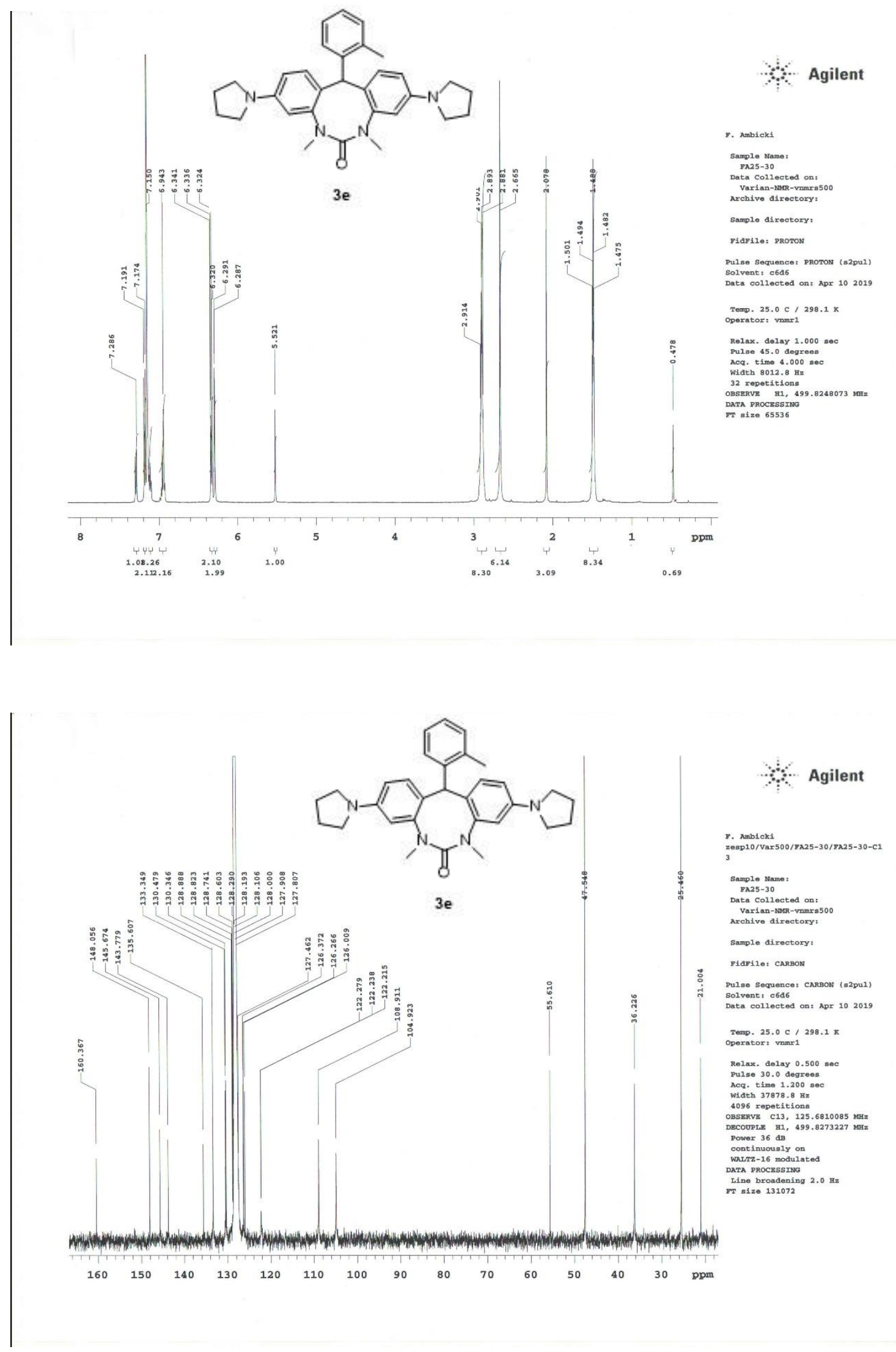

S18 

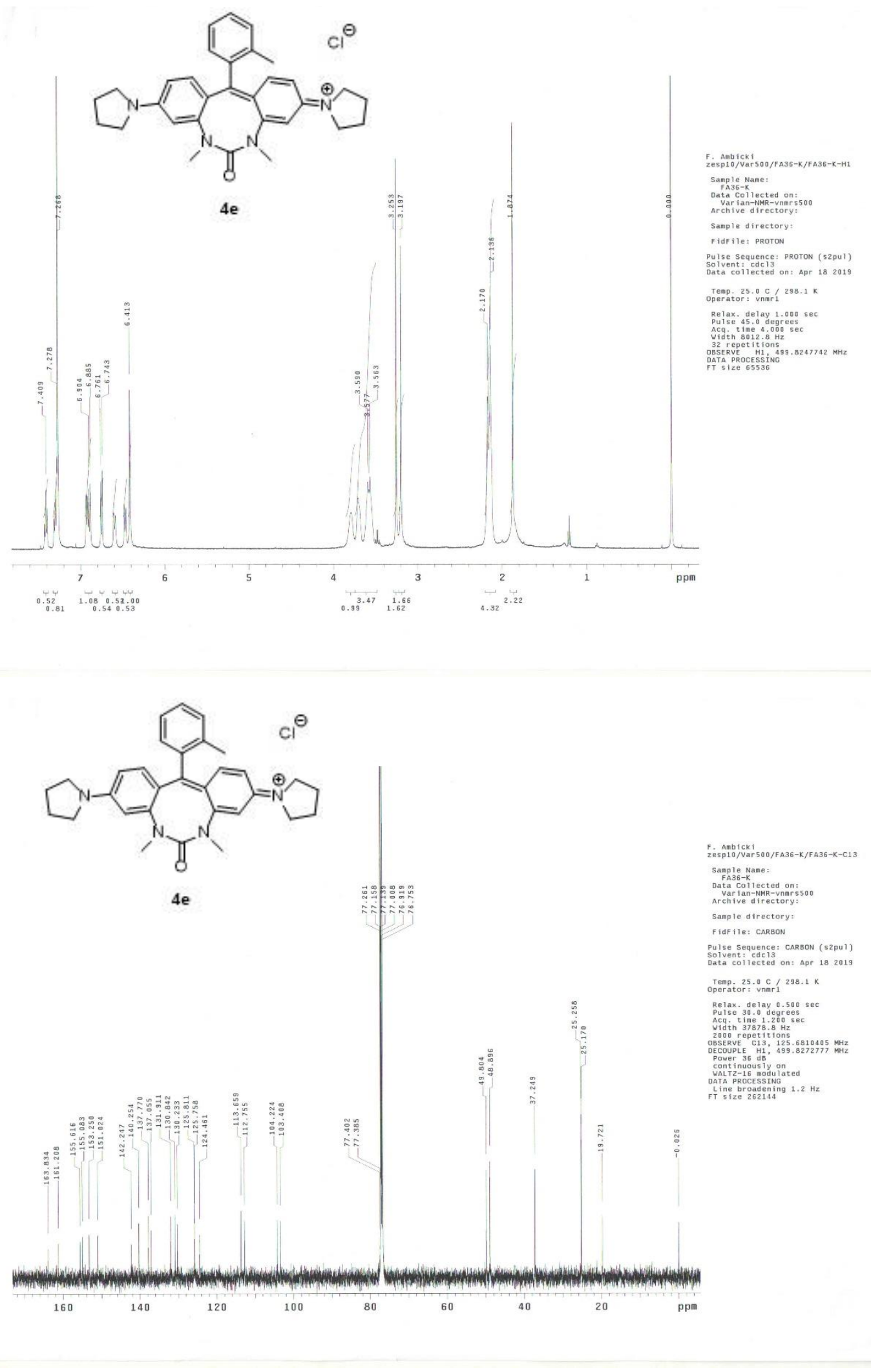


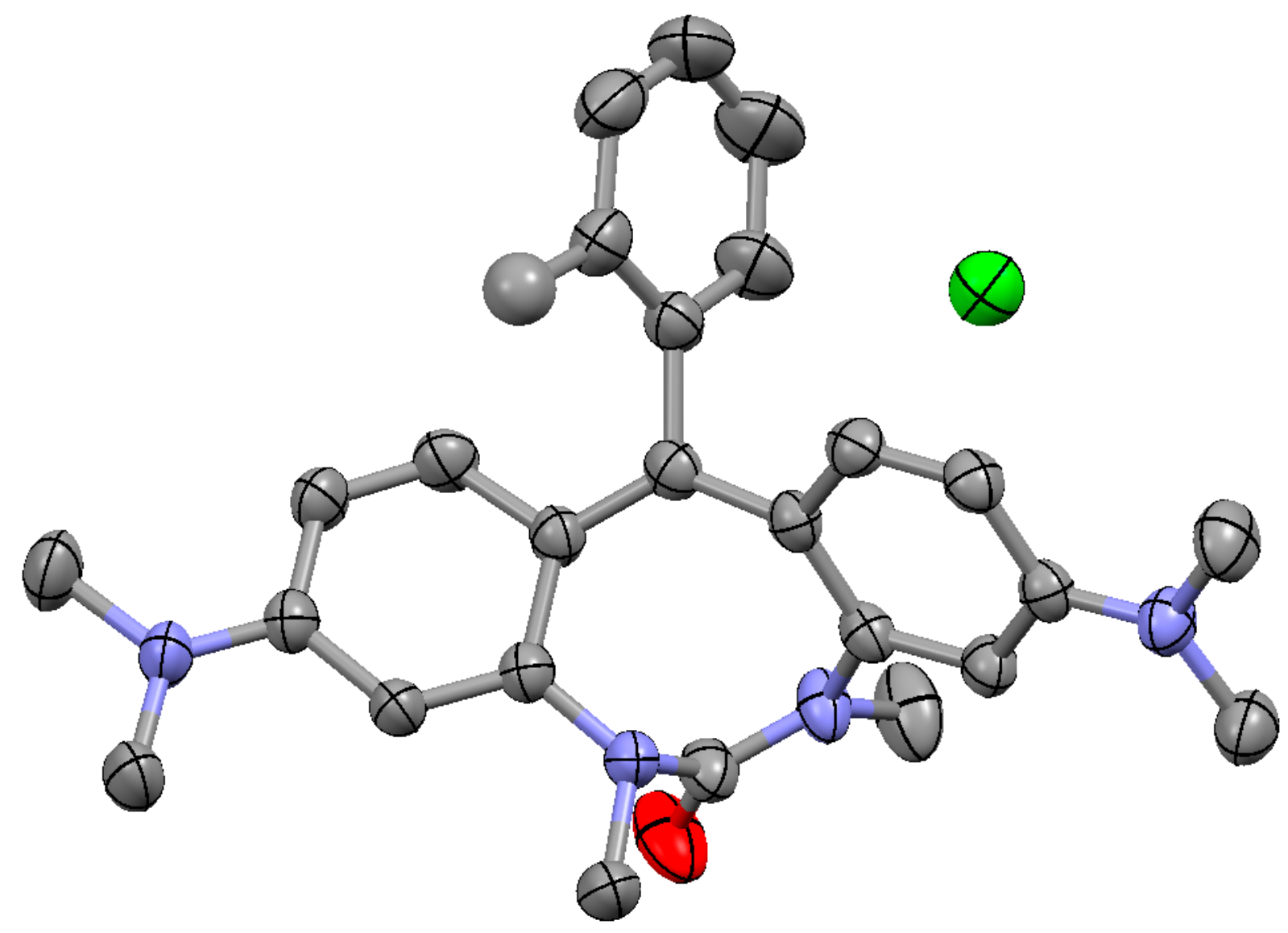

Figure S1. X-ray diffraction analysis for $\mathbf{4 b}$ (CCDC 1969445). The disorder of methyl groups of tolyl substituents is omitted for clarity. Ellipsoid probability levels $=50 \%$. X-ray crystallographic data:

monoclinic, P 21/c, a = 9.3250(2) $\AA, b=12.3792(3) \AA, c=22.7224(5) \AA ; \quad V=2621.09 \AA^{3}, Z=2 ; D_{c}=1.237 \mathrm{~g}$ $\mathrm{cm}^{-3} ; R_{1}=0.0511(I>2 \sigma(I)), w R_{2}=0.1310$ (all data), GOF $=1.046$. 


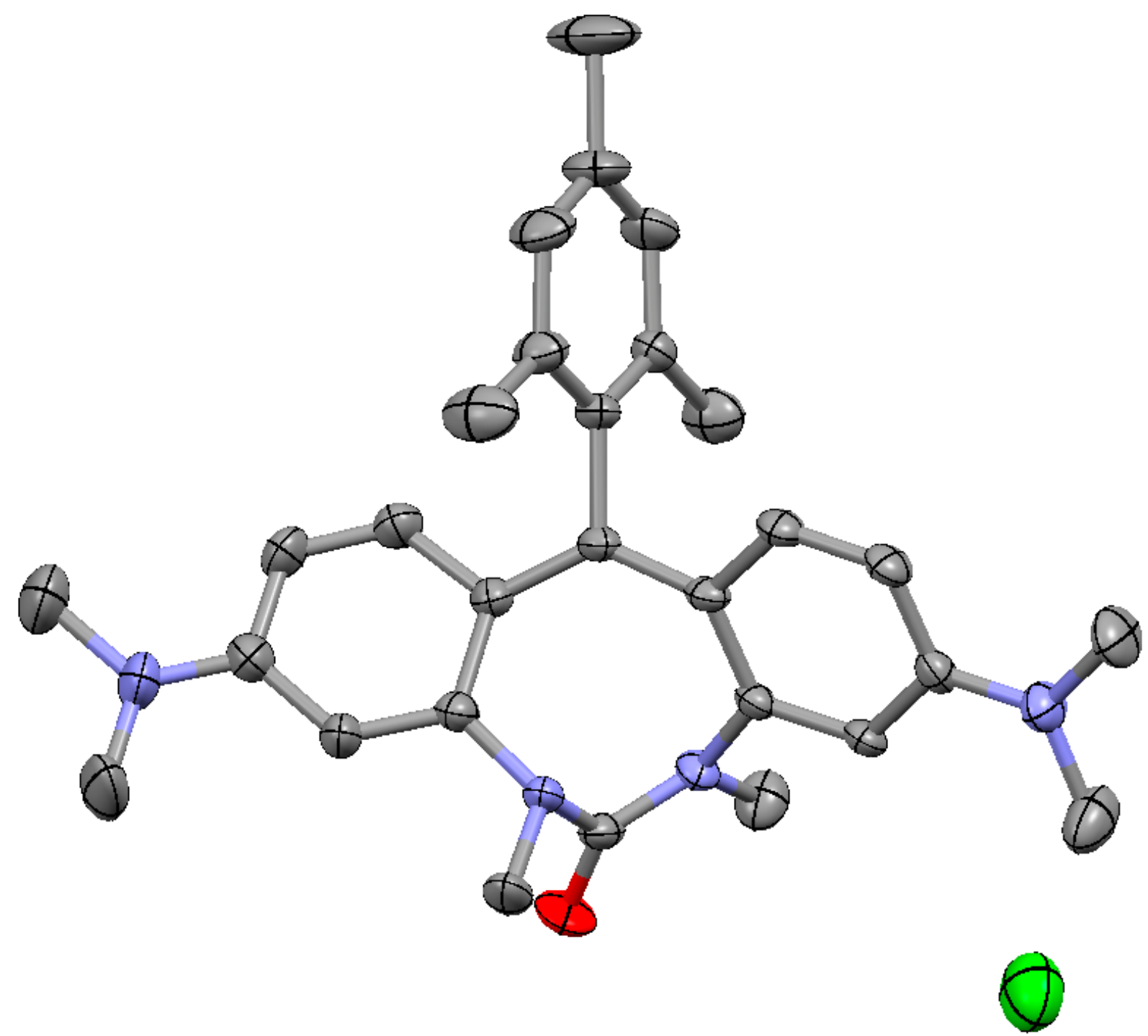

Figure S2. X-ray diffraction analysis for 4c (CCDC 1969446). Ellipsoid probability levels $=50 \%$. X-ray crystallographic data: triclinic, P $-1, a=9.5119(14) \AA, b=12.606(2) \AA, c=13.674(2) \AA$; $V=1520.4(4) \AA^{3}, Z$ $=2 ; D_{c}=1.073 \mathrm{~g} \mathrm{~cm}^{-3} ; R_{1}=0.1499(I>2 \sigma(I)), w R_{2}=0.3820$ (all data), GOF $=1.553$. Increased $R_{1}$ parameter is the result of the solvent disorder and is appropriately commented. 

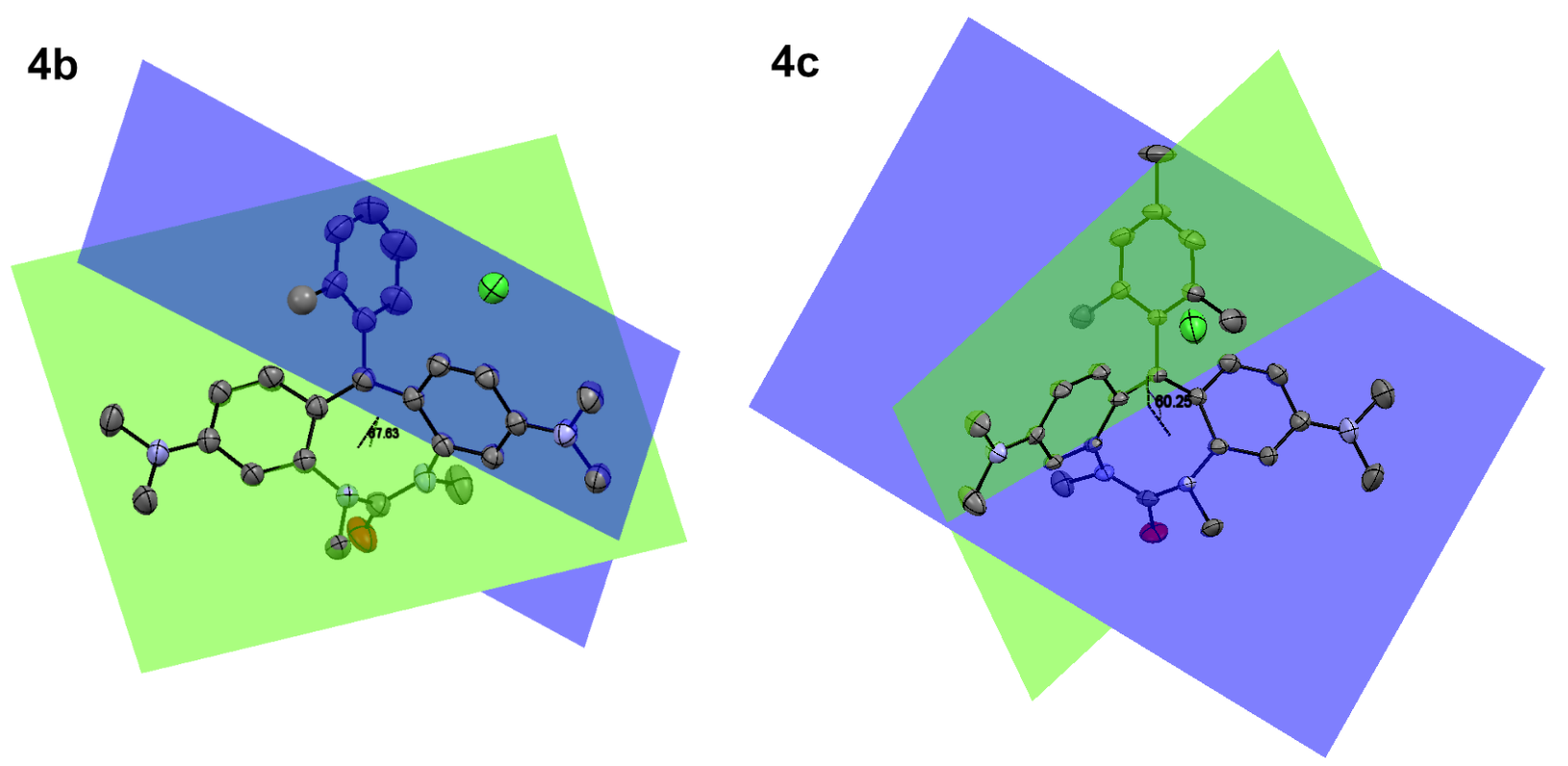

Figure S3. The dihedral angles between terminal aryl ring planes for compounds $\mathbf{4 b}$ and $\mathbf{4 c}$ based on $\mathrm{X}$ ray data. The disorder of methyl groups of tolyl substituents in $\mathbf{4 b}$ is omitted for clarity.
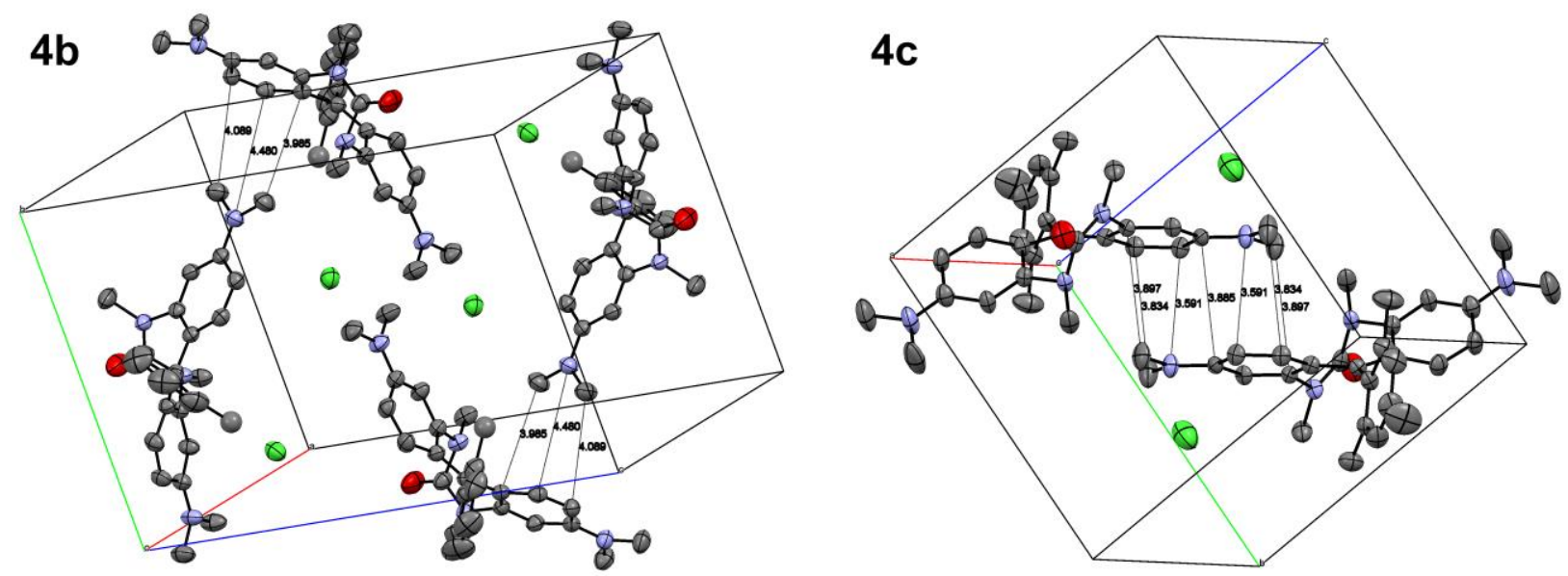

Figure S4. Crystal packing and intermolecular distances for compounds $\mathbf{4 b}$ and $\mathbf{4 c}$ based on X-ray data. The disorder of methyl groups of tolyl substituents in $\mathbf{4} \mathbf{b}$ is omitted for clarity. 


\section{Calculations}

All calculations were done with the Gaussian 09 package. ${ }^{1}$ DFT and TD DFT B3LYP/6-31G(d,p) methods were used for optimization of the ground $\left(S_{0}\right)$ and lowest excited $\left(S_{1}\right)$ electronic states of tested systems. The calculations were also repeated using the CAM-B3LYP/6-31G(d, p) method. The polarizable continuum model (PCM) with the default options of this model implemented in the Gaussian package was applied for the description of the solvent effect. The positive values of all calculated vibrations frequencies were confirmed the optimization of molecular structures in $\mathrm{S}_{0}$ state and occasionally structures in $\mathrm{S}_{1}$ state (limitation by the requirements of the computational time and memory). To minimize this deficiency, in the final of this supplement we present the full set of calculation results (with the optimization of structures in ground and excited electronic states in all forms postulated in our article) for Mini Model - a smaller molecule of $8 \mathrm{~m}$-rhodamine.

\section{Optimization of $8 \mathrm{U}-\mathrm{Rh}$ cations and comparison to the rhodamine B and malachite green structures}

Table S1. Comparison of the optimization results for $8 \mathrm{U}-\mathrm{Rh}(\mathbf{4 b})$, rhodamine $B(\mathrm{RB})$ and malachite green (MG) in their ground $S_{0}$ and electronic excited state $S_{1}$. DFT and TDDFT/B3LYP/6-31G(d,p) and CAM$B 3 L Y P / 6-31 G(d, p)$ calculations performed for cations placed in acetonitrile. In the subsequent lines: the optimized structures, HOMO and LUMO orbitals and characteristics of $S_{0} \rightarrow S_{1}$ and $S_{1} \rightarrow S_{0}$ transitions (energy in [eV] and [nm] and oscillator strength). HOMO and LUMO configuration dominates in the description of the $S_{0} \rightarrow S_{1}$ and $S_{1} \rightarrow S_{0}$ transitions.

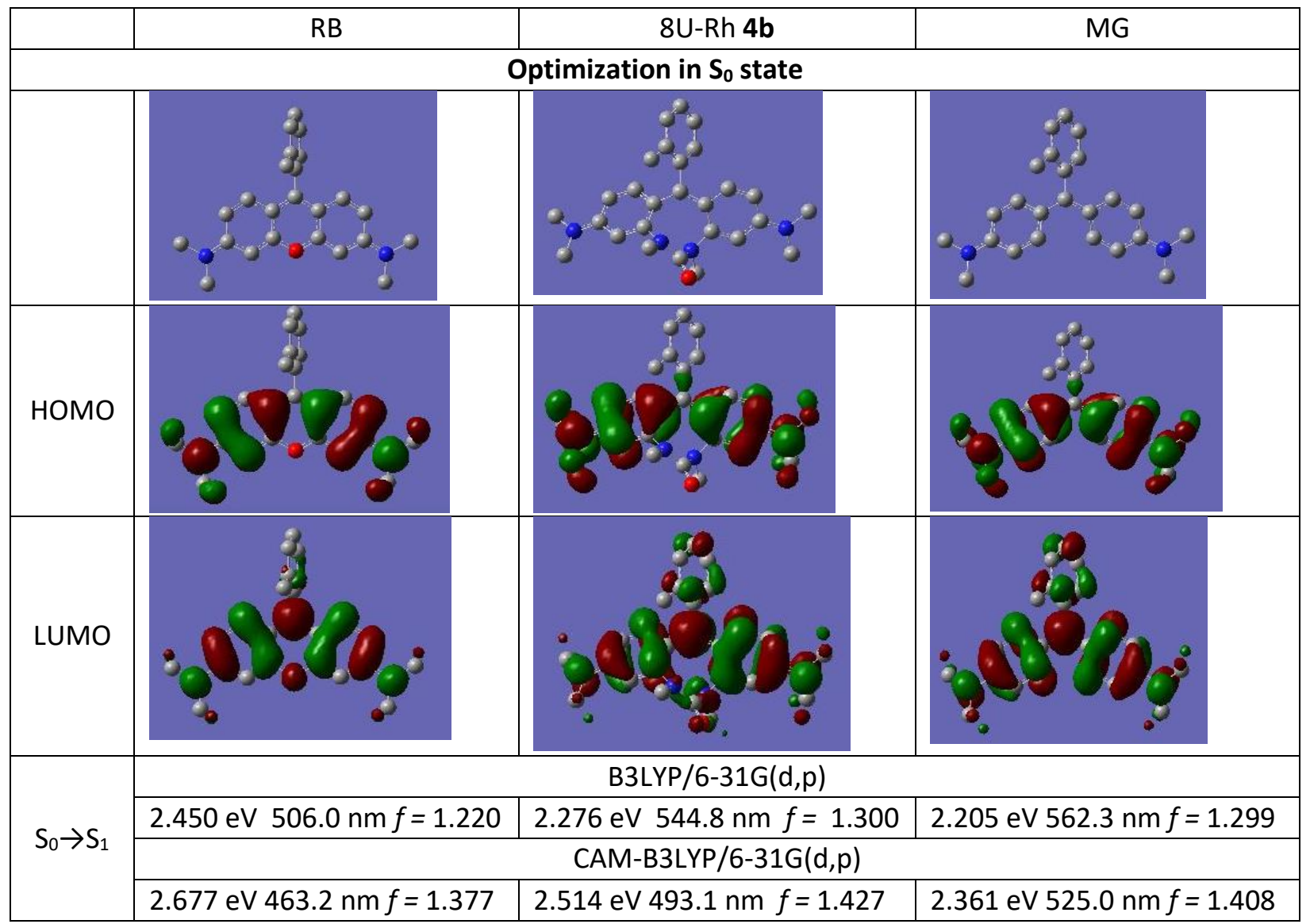




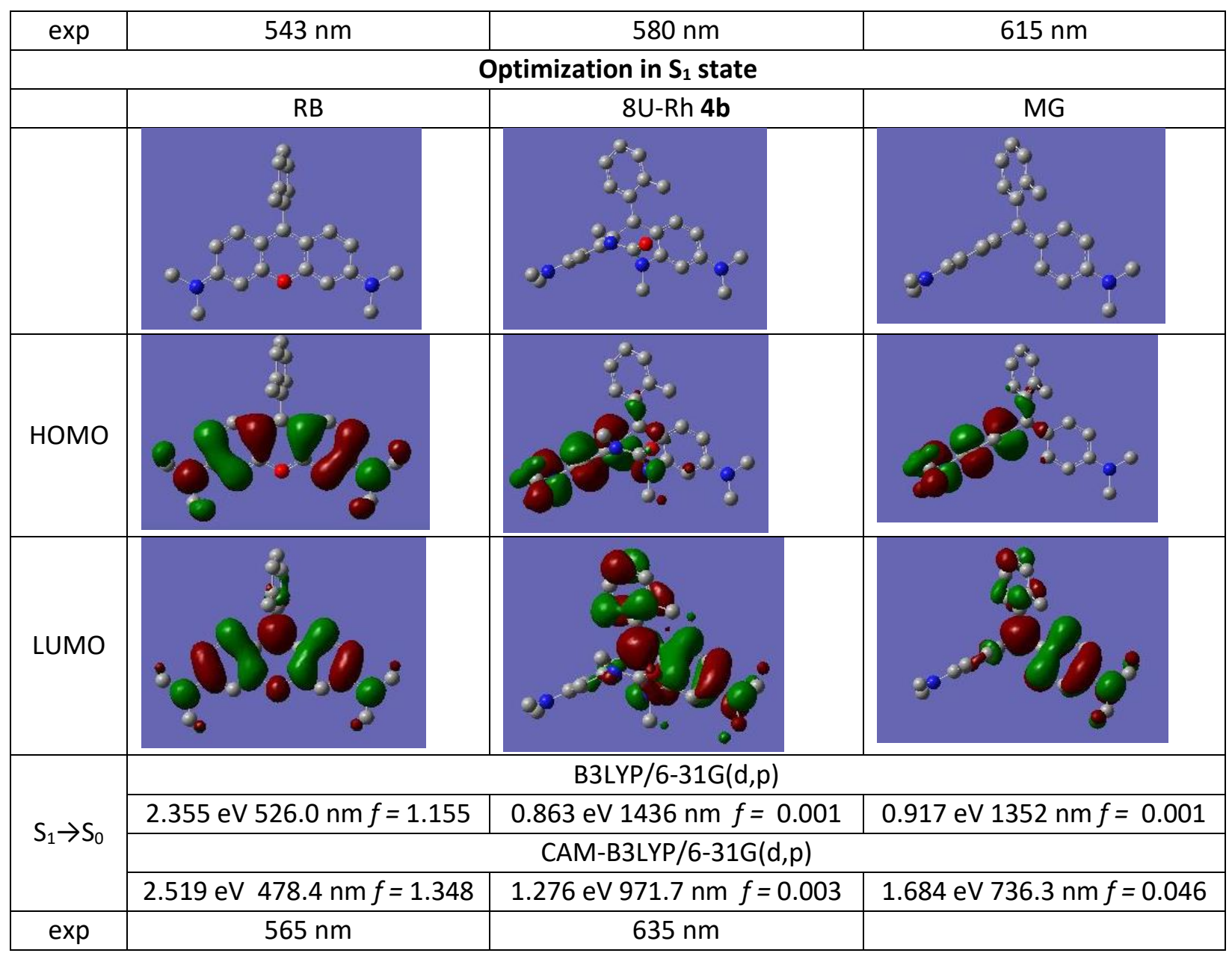

It is seen the large change of conformations of $\mathrm{MG}$ and $8 \mathrm{U}-\mathrm{Rh} \mathbf{4 b}$ cations optimized in excited electronic state in comparison to the ground state structure. It is also seen that transition $S_{1} \rightarrow S_{0}$ in MG and 8U-Rh $\mathbf{4 b}$ is of intramolecular charge transfer character. This results are confirmed within both calculations, by B3LYP/ and CAM-B3LYP/6-31G(d,p), i.e. both methods lead to qualitatively convergent results.

Experimental absorption energy values are better reproduced by the B3LYP method. In contrast, CT state energies calculated by B3LYP method are understated, which is a known feature of this method. From the point of view of the discussion in our work, it is important that it is a relatively low lying dark state. 
Table S2. Calculated solvent polarity effect on $S_{0} \rightarrow S_{1}$ transition of $\mathbf{4 b}$ cation (TD B3LYP/6-31G(d,p)).

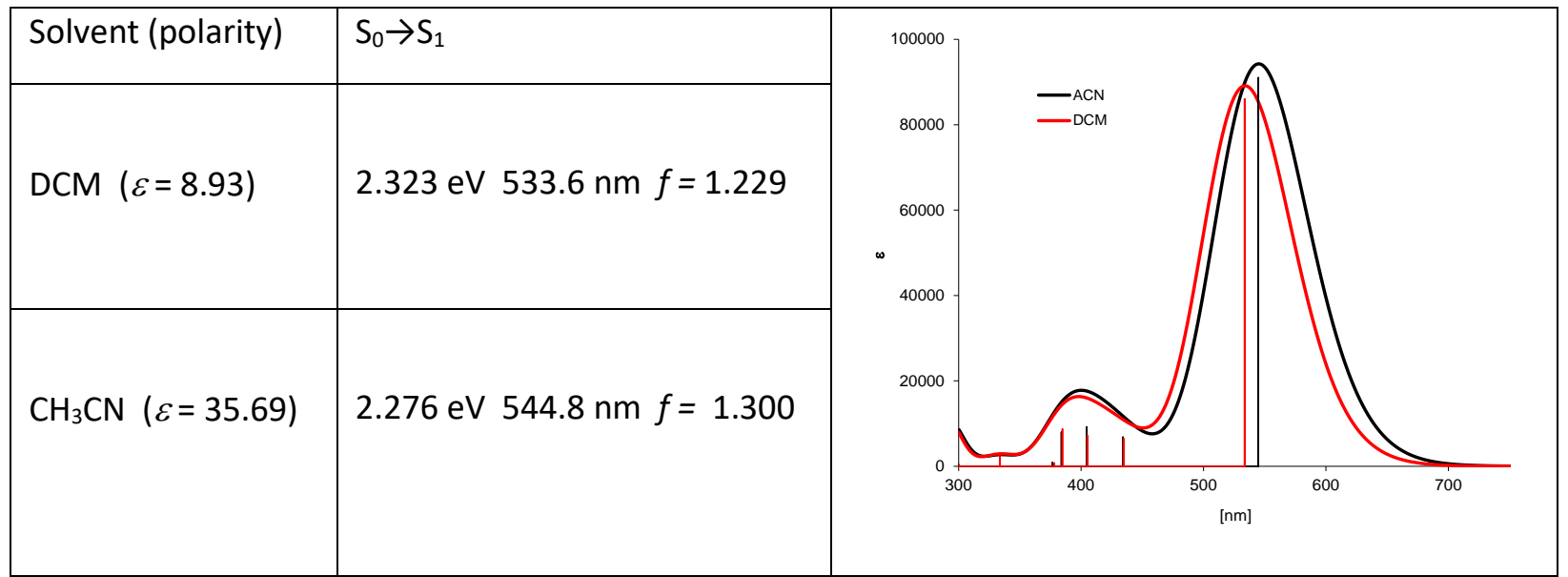

\section{Optimization of (8U-Rhs $\left.{ }^{+} \mathrm{Cl}^{-}\right)$ion pairs}

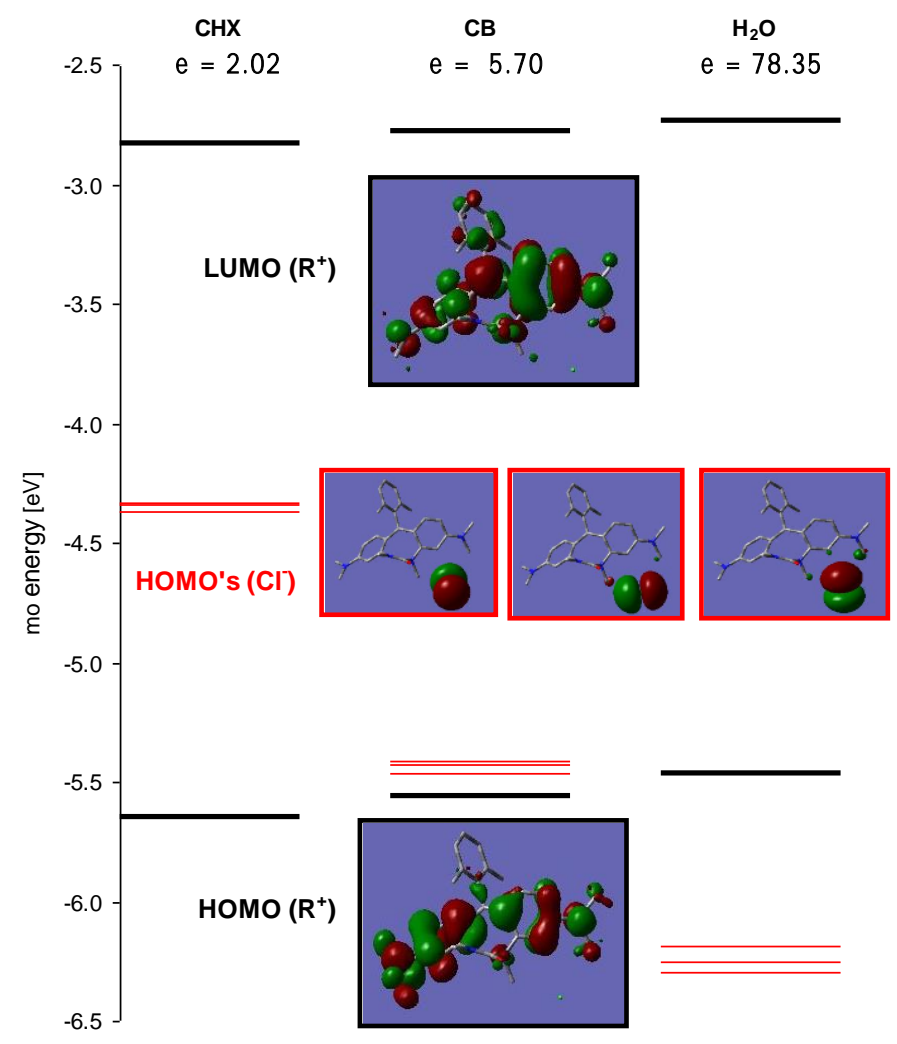

Figure S5. The ordering of molecular orbitals of $\mathbf{4 b}$ ion pair in solvents of different polarity. The levels corresponding to orbitals located on the $8 \mathrm{U}-\mathrm{Rh} \mathbf{4 b}$ cation are marked in black and the levels located on the $\mathrm{Cl}^{-}$anion are marked in red. For solvent of the large polarity the highest occupied molecular orbital of ion pair is $\mathrm{HOMO}\left(\mathrm{R}^{+}\right)$(localized on $\mathrm{R}^{+}$) while for solvent of low polarity highest orbital of ion pair is located on anion $\mathrm{Cl}^{-}(\mathrm{B} 3 \mathrm{LYP} / 6-31 \mathrm{G}(\mathrm{d}, \mathrm{p}))$. 
Table S3. Energies of $\mathrm{S}_{0} \rightarrow S_{i}$ for ion pair $\mathrm{R}^{+} \mathrm{Cl}^{-}$of $\mathbf{4 b}$ in solvents of different polarity. For solvent of high polarity $\left(\mathrm{CH}_{3} \mathrm{CN}\right) \mathrm{S}_{0} \rightarrow \mathrm{S}_{1}$ transition is determined by $\mathrm{HOMO}\left(\mathrm{R}^{+}\right) \rightarrow \mathrm{LUMO}\left(\mathrm{R}^{+}\right) \mathrm{LE}$ configuration localized on cation $\mathrm{R}^{+}$(see Fig.S3) but for solvent of low polarity (CB) $\mathrm{S}_{0} \rightarrow \mathrm{S}_{1}$ transition is determined by charge transfer configuration $\mathrm{HOMO}\left(\mathrm{Cl}^{-}\right) \rightarrow \mathrm{LUMO}\left(\mathrm{R}^{+}\right)$(see Fig.S3) (TD B3LYP/6-31G(d,p) calculations).

\begin{tabular}{|c|c|c|}
\hline \multicolumn{3}{|c|}{ CB $\varepsilon=5.697$} \\
\hline $\mathrm{R}^{+}$ & $S_{1} \quad 2.4696 \mathrm{eV} 502.04 \mathrm{~nm} f=0.7782$ & \\
\hline $\mathrm{R}^{+} \mathrm{Cl}^{-}$ & $\begin{array}{ll}\mathrm{S}_{1} & 2.2191 \mathrm{eV} 558.72 \mathrm{~nm} f=0.0182 \mathrm{CT} \\
\mathrm{S}_{2} & 2.2421 \mathrm{eV} 552.98 \mathrm{~nm} f=0.0120 \mathrm{CT} \\
\mathrm{S}_{3} & 2.2512 \mathrm{eV} 550.74 \mathrm{~nm} f=0.0014 \mathrm{CT} \\
\mathrm{S}_{4} & \mathbf{2 . 5 1 7 0 ~ e V ~} \mathbf{4 9 2 . 5 9} \mathbf{n m} \boldsymbol{f}=\mathbf{0 . 7 6 2 5} \mathrm{LE}\end{array}$ & 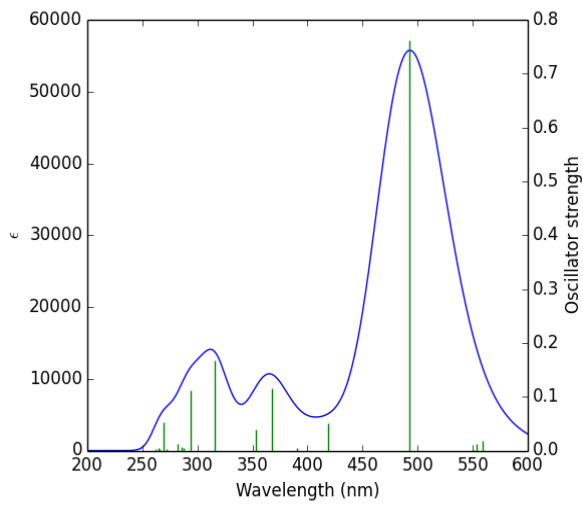 \\
\hline \multicolumn{3}{|c|}{ DCM $\varepsilon=8.93$} \\
\hline $\mathrm{R}^{+}$ & $\mathrm{S}_{1} 2.4835 \mathrm{eV} 499.23 \mathrm{~nm} f=0.7538$ & \\
\hline $\mathrm{R}^{+} \mathrm{Cl}^{-}$ & $\begin{array}{l}\mathrm{S}_{1} 2.4508 \mathrm{eV} 505.90 \mathrm{~nm} f=0.2433 \mathrm{LE}+\mathrm{CT} \\
\mathrm{S}_{2} 2.4902 \mathrm{eV} 497.89 \mathrm{~nm} f=0.0726 \mathrm{CT}+\mathrm{LE} \\
\mathrm{S}_{3} 2.5116 \mathrm{eV} 493.64 \mathrm{~nm} f=0.0144 \mathrm{CT}+\mathrm{LE} \\
\mathrm{S}_{4} 2.5473 \mathrm{eV} 486.73 \mathrm{~nm} f=0.4341 \mathrm{LE}+\mathrm{CT}\end{array}$ & (20) \\
\hline \multicolumn{3}{|c|}{$\mathrm{CH}_{3} \mathrm{CN} \quad \varepsilon=35.688$} \\
\hline $\mathrm{R}^{+}$ & $S_{1} \quad 2.4952 \mathrm{eV} 496.90 \mathrm{~nm} f=0.7326$ & \\
\hline $\mathrm{R}^{+} \mathrm{Cl}^{-}$ & 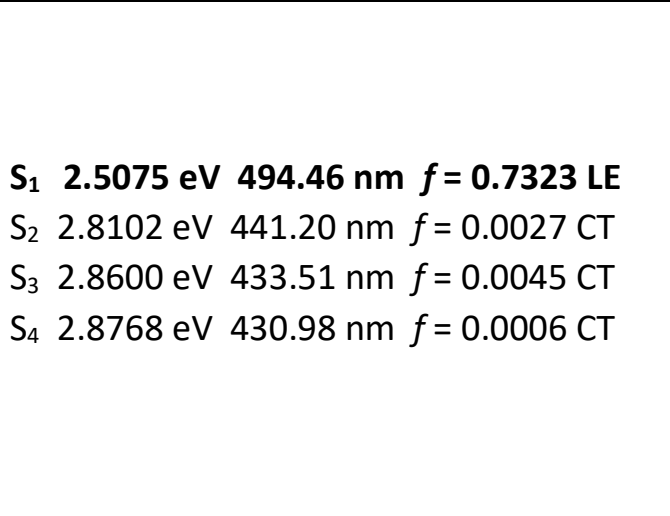 & (20) \\
\hline
\end{tabular}


Table S4. Ground $\left(S_{0}\right)$ and excited state $\left(S_{1}-A\right.$ and $\left.S_{1}-B\right)$ structures of ion pair $8 \mathrm{U}-\mathrm{Rh}^{+} \mathrm{Cl}^{-} \mathbf{4 b}$ in acetonitrile. $\mathrm{HOMO}$ and LUMO orbitals and data on transitions energies and oscillator strengths are given. $\mathrm{R}(\mathrm{C}-\mathrm{Cl})$ is the distance between $\mathrm{Cl}$ and $\mathrm{C}$ - central $\mathrm{C}$ atom of dye chromophore given in [Å] (results of B3LYP and TD B3LYP/6-31G(d,p) calculations).

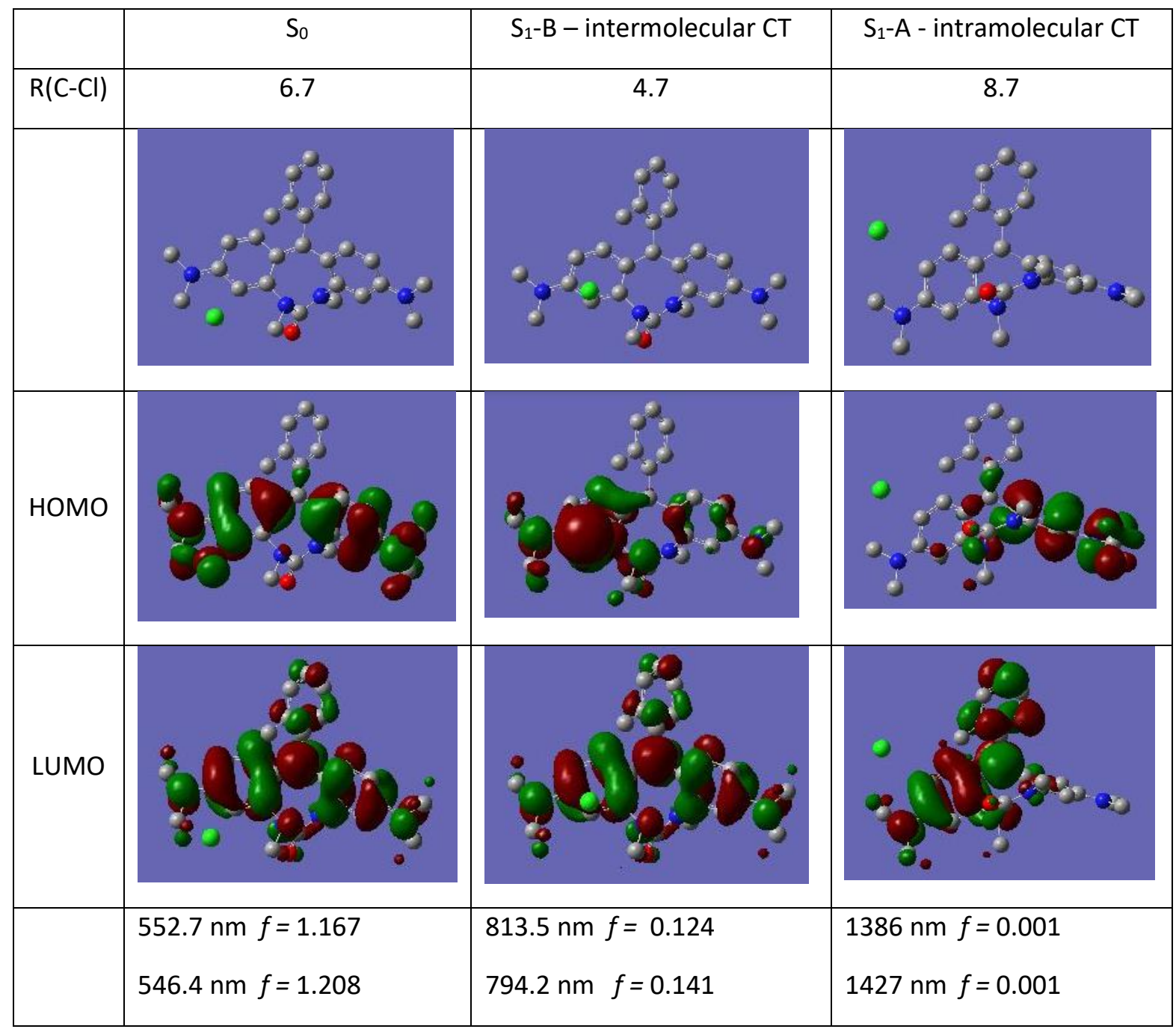


Table S5a. Coordinates of $\mathbf{4 b}$ cation optimized in $\mathrm{S}_{0}$ and $\mathrm{S}_{1}$ states - structures shown in Table $\mathrm{S} 1$.

\begin{tabular}{|c|c|c|c|c|c|c|c|}
\hline & & & & $\mathrm{S}_{1}$ & & & \\
\hline & 0.4982 & -3.4264 & 1.4808 & 0 & 0.7485 & -0.3315 & 3.0572 \\
\hline & 0.6183 & -2.0386 & -0.3984 & $\mathrm{~N}$ & 0.5391 & -1.4848 & 1.0174 \\
\hline & -0.8994 & -1.6252 & 1.3518 & $\mathrm{~N}$ & -1.2551 & -0.2768 & 1.9404 \\
\hline & 5.3214 & -0.9337 & -0.5164 & $\mathrm{~N}$ & 5.0577 & -1.7247 & -0.8203 \\
\hline & -5.1719 & -1.0635 & -0.8067 & $\mathrm{~N}$ & -4.8576 & -1.8727 & -0.8763 \\
\hline & 0.0826 & -2.4449 & 0.8873 & $C$ & 0.0640 & -0.6273 & 2.0907 \\
\hline & 1.6460 & -1.0854 & -0.2310 & $\mathrm{C}$ & 1.6014 & -0.9603 & 0.2435 \\
\hline & 2.9580 & -1.4356 & -0.5033 & $C$ & 2.8110 & -1.6226 & 0.1003 \\
\hline & 4.0518 & -0.5688 & -0.2345 & $\mathrm{C}$ & 3.8727 & -1.0564 & -0.6556 \\
\hline & 3.7616 & 0.6810 & 0.4016 & $C$ & 3.6681 & 0.2344 & -1.2152 \\
\hline & 2.4669 & 1.0645 & 0.5896 & $C$ & 2.4578 & 0.8827 & -1.0681 \\
\hline & 1.3305 & 0.2648 & 0.2089 & C & 1.3630 & 0.3065 & -0.3779 \\
\hline & 0.0802 & 0.9085 & 0.0565 & $C$ & 0.0608 & 0.9137 & -0.3501 \\
\hline & -1.2099 & 0.3118 & -0.1791 & C & -1.1376 & 0.0256 & -0.4749 \\
\hline & -2.1442 & 1.0092 & -1.0018 & $\mathrm{C}$ & -1.6487 & -0.3173 & -1.7439 \\
\hline & -3.4247 & 0.5713 & -1.2309 & C & -2.8630 & -0.9517 & -1.8930 \\
\hline & -3.9063 & -0.6159 & -0.6060 & C & -3.6409 & -1.2957 & -0.7420 \\
\hline & -2.9978 & -1.3156 & 0.2325 & C & & & 504 \\
\hline & -1.6971 & -0.8832 & 0.4238 & C & -1.8586 & -0.4429 & 0.6713 \\
\hline & 0.0898 & 2.4077 & 0.0192 & C & -0.1708 & 2.3567 & -0.3702 \\
\hline & 0.7834 & 3.0550 & -1.0191 & C & -1.2749 & 2.8850 & -1.0923 \\
\hline & 0.7808 & 4.4435 & -1.1304 & C & -1.5385 & 4.2 & -1.1595 \\
\hline & 0.1045 & 5.2045 & -0.1771 & C & -0.7093 & 5.1460 & -0.4880 \\
\hline & -0.5663 & 4.5707 & 0.8697 & C & 0.3556 & 4.6506 & 0.2653 \\
\hline & -0.6037 & 3.1753 & 0.9856 & C & 0.6443 & 3.2847 & 0.3601 \\
\hline$C$ & 0.8759 & -3.1912 & -1.2762 & C & 0.5697 & -2.8923 & 1.4257 \\
\hline & -1.4365 & -1.9116 & 2.6894 & C & -1.9698 & 0.4091 & 3.0217 \\
\hline & 6.4482 & -0.0814 & -0.1259 & C & 6.1763 & -1.0780 & -1.4972 \\
\hline & 5.6039 & -2.2124 & -1.1716 & C & 5.2504 & -3.0443 & -0.2318 \\
\hline C & -5.6428 & -2.2797 & -0.1459 & C & -5.7685 & -2.0373 & 0.2600 \\
\hline & -6.0835 & -0.3345 & -1.6881 & C & -5.3506 & -2.3725 & -2.1644 \\
\hline & -1.3441 & 2.5452 & 2.1439 & C & 1.7540 & 2.8693 & 1.3007 \\
\hline & 4.5603 & 1.3368 & 0.7199 & $\mathrm{H}$ & 4.4477 & 0.7099 & -1.7955 \\
\hline H & 2.2783 & 2.0281 & 1.0451 & $\mathrm{H}$ & 2.3135 & 1.8421 & -1.5543 \\
\hline 7 & -4.0623 & 1.1410 & -1.8935 & $\mathrm{H}$ & -3.2467 & -1.1345 & -2.8870 \\
\hline & -3.3141 & -2.2181 & 0.7361 & $\mathrm{H}$ & -3.5933 & -1.4023 & 1.4377 \\
\hline & 1.3074 & 4.9235 & -1.9491 & $\mathrm{H}$ & -2.3853 & 4.6024 & -1.7390 \\
\hline & 0.1033 & 6.2884 & -0.2414 & $\mathrm{H}$ & -0.8986 & 6.2141 & -0.5301 \\
\hline & -1.0743 & 5.1706 & 1.6199 & $\mathrm{H}$ & 0.9747 & 5.3460 & 0.8265 \\
\hline & 1.3100 & 2.4542 & -1.7539 & $\mathrm{H}$ & -1.9238 & 2.2086 & -1.6357 \\
\hline
\end{tabular}




$\begin{array}{llllllll}\text { H } & 1.2376 & -2.8226 & -2.2368 & \text { H } & 0.7587 & -3.5149 & 0.5492 \\ \text { H } & -0.0706 & -3.7113 & -1.4350 & \text { H } & -0.4075 & -3.1585 & 1.8361 \\ \text { H } & 1.5949 & -3.9022 & -0.8567 & \text { H } & 1.3340 & -3.0970 & 2.1872 \\ \text { H } & -2.0838 & -2.7944 & 2.6931 & \text { H } & -2.8225 & -0.1843 & 3.3616 \\ \text { H } & -2.0002 & -1.0442 & 3.0315 & \text { H } & -2.3215 & 1.3858 & 2.6808 \\ \text { H } & -0.6010 & -2.0955 & 3.3652 & \text { H } & -1.2726 & 0.5394 & 3.8468 \\ \text { H } & 6.3831 & 0.9005 & -0.6046 & \text { H } & 5.9330 & -0.8406 & -2.5396 \\ \text { H } & 7.3745 & -0.5559 & -0.4426 & \text { H } & 7.0310 & -1.7527 & -1.4962 \\ \text { H } & 6.4855 & 0.0554 & 0.9596 & \text { H } & 6.4726 & -0.1497 & -0.9939 \\ \text { H } & 6.6592 & -2.2497 & -1.4342 & \text { H } & 6.2129 & -3.4403 & -0.5523 \\ \text { H } & 5.0182 & -2.3148 & -2.0895 & \text { H } & 4.4718 & -3.7415 & -0.5604 \\ \text { H } & 5.3797 & -3.0591 & -0.5127 & \text { H } & 5.2418 & -3.0116 & 0.8657 \\ \text { H } & -5.0579 & -3.1540 & -0.4534 & \text { H } & -5.5739 & -2.9870 & 0.7691 \\ \text { H } & -6.6823 & -2.4514 & -0.4183 & \text { H } & -6.7897 & -2.0446 & -0.1190 \\ \text { H } & -5.5857 & -2.1874 & 0.9442 & \text { H } & -5.6624 & -1.2124 & 0.9619 \\ \text { H } & -6.2766 & 0.6773 & -1.3151 & \text { H } & -5.8387 & -1.5673 & -2.7225 \\ \text { H } & -7.0311 & -0.8676 & -1.7355 & \text { H } & -6.0796 & -3.1574 & -1.9687 \\ \text { H } & -5.6807 & -0.2619 & -2.7041 & \text { H } & -4.5385 & -2.7928 & -2.7544 \\ \text { H } & -1.8156 & 1.9149 & -1.4967 & \text { H } & -1.0910 & -0.0258 & -2.6266 \\ \text { H } & 3.1618 & -2.4312 & -0.8646 & \text { H } & 2.9607 & -2.5604 & 0.6153 \\ \text { H } & -1.5216 & 3.2837 & 2.9295 & \text { H } & 1.9447 & 3.6691 & 2.0215 \\ \text { H } & -0.7852 & 1.7130 & 2.5809 & \text { H } & 2.6951 & 2.6670 & 0.7788 \\ \text { H } & -2.3186 & 2.1540 & 1.8319 & \text { H } & 1.5007 & 1.9630 & 1.8566\end{array}$

Table S5b. Coordinates of ion pair $\mathbf{4} \mathbf{b}^{+} \mathrm{Cl}^{-}$optimized in $\mathrm{S}_{0}, \mathrm{~S}_{1}-\mathrm{A}$ and $\mathrm{S}_{1}-\mathrm{B}$ states - structures shown in Table S4.

\begin{tabular}{llllllllllll}
$\mathrm{S}_{0}$ & \multicolumn{10}{c}{$\mathrm{S}-\mathrm{A}$} \\
$\mathrm{Cl}$ & -3.7415 & -3.0693 & 3.1130 & $\mathrm{Cl}$ & -6.2300 & 1.3969 & -2.7004 & $\mathrm{Cl}$ & -2.7990 & -0.6798 & 2.8691 \\
$\mathrm{O}$ & -0.4440 & -2.5458 & -2.6743 & $\mathrm{O}$ & 0.3351 & -0.9691 & 3.2366 & $\mathrm{O}$ & -0.2890 & -3.2772 & -1.8357 \\
$\mathrm{~N}$ & -0.6355 & -1.7277 & -0.4903 & $\mathrm{~N}$ & 5.2382 & -1.1748 & -1.7866 & $\mathrm{~N}$ & -0.4769 & -1.9673 & 0.0874 \\
$\mathrm{~N}$ & 1.1736 & -1.1465 & -1.8811 & $\mathrm{~N}$ & 0.2732 & -1.7744 & 1.0262 & $\mathrm{~N}$ & 1.1545 & -1.5306 & -1.5620 \\
$\mathrm{~N}$ & -5.1302 & 0.0274 & -0.3376 & $\mathrm{~N}$ & 2.0799 & -0.4991 & 1.8227 & $\mathrm{~N}$ & -5.1868 & -0.8290 & -0.1280 \\
$\mathrm{~N}$ & 5.0846 & -1.8052 & 0.8616 & $\mathrm{~N}$ & -4.4532 & -2.3451 & -0.0724 & $\mathrm{~N}$ & 5.3443 & -1.2169 & 0.8344 \\
$\mathrm{C}$ & 0.0205 & -1.8579 & -1.7796 & $\mathrm{C}$ & -1.3140 & 2.2882 & 2.2256 & $\mathrm{C}$ & 0.1294 & -2.3314 & -1.1816 \\
$\mathrm{C}$ & -1.5038 & -0.6204 & -0.4717 & $\mathrm{C}$ & -4.4118 & -3.7218 & 0.4044 & $\mathrm{C}$ & -1.4582 & -0.9646 & -0.0835 \\
$\mathrm{C}$ & -2.8698 & -0.8186 & -0.3711 & $\mathrm{C}$ & -5.7644 & -1.7461 & -0.3115 & $\mathrm{C}$ & -2.8149 & -1.3191 & 0.0737 \\
$\mathrm{C}$ & -3.8058 & 0.2455 & -0.4716 & $\mathrm{C}$ & 6.3523 & -1.3987 & -0.8609 & $\mathrm{C}$ & -3.8855 & -0.4491 & -0.2999 \\
$\mathrm{C}$ & -3.2934 & 1.5481 & -0.7836 & $\mathrm{C}$ & 0.4691 & -3.2183 & 1.1843 & $\mathrm{C}$ & -3.5205 & 0.7816 & -0.8811 \\
$\mathrm{C}$ & -1.9483 & 1.7636 & -0.7943 & $\mathrm{C}$ & 2.9215 & 0.0880 & 2.8699 & $\mathrm{C}$ & -2.1875 & 1.1544 & -0.9389 \\
$\mathrm{C}$ & -0.9701 & 0.7354 & -0.5339 & $\mathrm{C}$ & -3.3369 & -1.5504 & 0.0162 & $\mathrm{C}$ & -1.0967 & 0.3662 & -0.4756 \\
$\mathrm{C}$ & 0.3173 & 1.1294 & -0.1121 & $\mathrm{C}$ & -2.1034 & -2.0728 & 0.4907 & $\mathrm{C}$ & 0.1879 & 1.0016 & -0.2544 \\
$\mathrm{C}$ & 1.4775 & 0.2996 & 0.1170 & $\mathrm{C}$ & -3.3737 & -0.1760 & -0.3487 & $\mathrm{C}$ & 1.4579 & 0.3677 & 0.0127
\end{tabular}




\begin{tabular}{|c|c|c|c|c|c|c|c|c|c|c|c|}
\hline & & & & C & & & & & & & \\
\hline & & 473 & & C & & & & & & & \\
\hline & & & & $c$ & & & & & & & \\
\hline & & & & C & & & & & & & \\
\hline & & & & C & & & & & & & \\
\hline & & & & & & & & & & & \\
\hline & & & & ( & & & & & & & \\
\hline & .0573 & & & 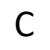 & & & & & & & \\
\hline & & & & ( & & & & & & & \\
\hline & & & & ( & & & & & & & \\
\hline & & & & C & & & & & & & \\
\hline & 61 & & & C & & & & & & & \\
\hline & 2 & 39 & & C & & & & & & & \\
\hline & 7 & & & C & & & & & & & \\
\hline & & & & & & & & & & & \\
\hline & 1 & & & C & & & & & & & \\
\hline & 41 & 51 & 8 & ( & & & & & & & 304 \\
\hline & & & & C & & & & & & & \\
\hline & & & & $\mathrm{H}$ & & & & & & & \\
\hline & & & & $\mathrm{H}$ & & & & & & & \\
\hline & 79 & 0 & & $\mathrm{H}$ & & & & & & & \\
\hline & & & & $\mathrm{H}$ & & & & & & & \\
\hline & 0.6 & .74 & 08 & $\mathrm{H}$ & & & & H & & & 883 \\
\hline & & & & $\mathrm{H}$ & & & & & & & \\
\hline & & & & $\mathrm{H}$ & & & & & & & \\
\hline & 30 & & & $\mathrm{H}$ & & & & & & & \\
\hline & 60 & 84 & 64 & $\mathrm{H}$ & & & & $\mathrm{H}$ & & 998 & 8558 \\
\hline & & & & $\mathrm{H}$ & & & & & & & \\
\hline & & & & $\mathrm{H}$ & & & & $\mathrm{H}$ & & & \\
\hline & & 87 & & $\mathrm{H}$ & & & & $\mathrm{H}$ & & & \\
\hline & 6014 & 48 & 35 & $\mathrm{H}$ & 64 & & & $\mathrm{H}$ & & 07 & -3 \\
\hline & & & & $\mathrm{H}$ & & & & & & & \\
\hline & & & & $\mathrm{H}$ & & & & & & & \\
\hline & & & & $\mathrm{H}$ & & & & & & & \\
\hline & -6.0052 & 4795 & 71 & $\mathrm{H}$ & & & & & & & 660 \\
\hline & -6.6918 & 112 & & $\mathrm{H}$ & & & & & & & 007 \\
\hline & & & & $\mathrm{H}$ & & & & & & & \\
\hline & & & & $\mathrm{H}$ & & & & & & & \\
\hline & .7335 & -3.7191 & .0144 & $\mathrm{H}$ & 5.8584 & & & $\mathrm{H}$ & & & .5990 \\
\hline & & -3 & & $\mathrm{H}$ & & & & & & & 491 \\
\hline & & & & $\mathrm{H}$ & & & & & & 34 & -0.8 \\
\hline & & & & $\mathrm{H}$ & & & & & & & 1.5670 \\
\hline & & -2.1031 & 0104 & $\mathrm{H}$ & & -1.1368 & 0.5394 & П & 7.1458 & -1.1222 & 1.06 \\
\hline
\end{tabular}


$\begin{array}{llllllllllll}H & 5.4018 & -1.5608 & 2.9467 & H & -5.7583 & -1.1109 & -1.2013 & H & 5.7467 & -0.5959 & 2.8312 \\ H & 2.0694 & 1.4424 & 1.8529 & H & -4.2825 & -3.7864 & 1.4941 & H & 2.0703 & 1.9047 & 1.4001 \\ H & -3.2396 & -1.8269 & -0.2774 & H & -5.3471 & -4.2154 & 0.1417 & H & -3.0426 & -2.3363 & 0.3516 \\ H & 2.6023 & 3.7888 & -2.1593 & H & -3.5965 & -4.2746 & -0.0720 & H & 1.9923 & 3.5455 & -2.8398 \\ H & 1.5916 & 2.3422 & -2.2912 & H & 0.6239 & 6.1809 & 0.5913 & H & 1.2604 & 1.9450 & -2.6093 \\ H & 3.0498 & 2.3110 & -1.3024 & H & -1.4571 & 2.9418 & 3.0905 & H & 2.7249 & 2.3756 & -1.7367\end{array}$ 


\section{Mini Model}

Computation data for $8 \mathrm{U}-\mathrm{Rh}$ simplified model are listed below:

Table S6. HOMO and LUMO orbitals of $\mathrm{R}^{+}$optimized in $\mathrm{S}_{0}$ and $\mathrm{S}_{1}$ electronic state and data on the electronic transitions.

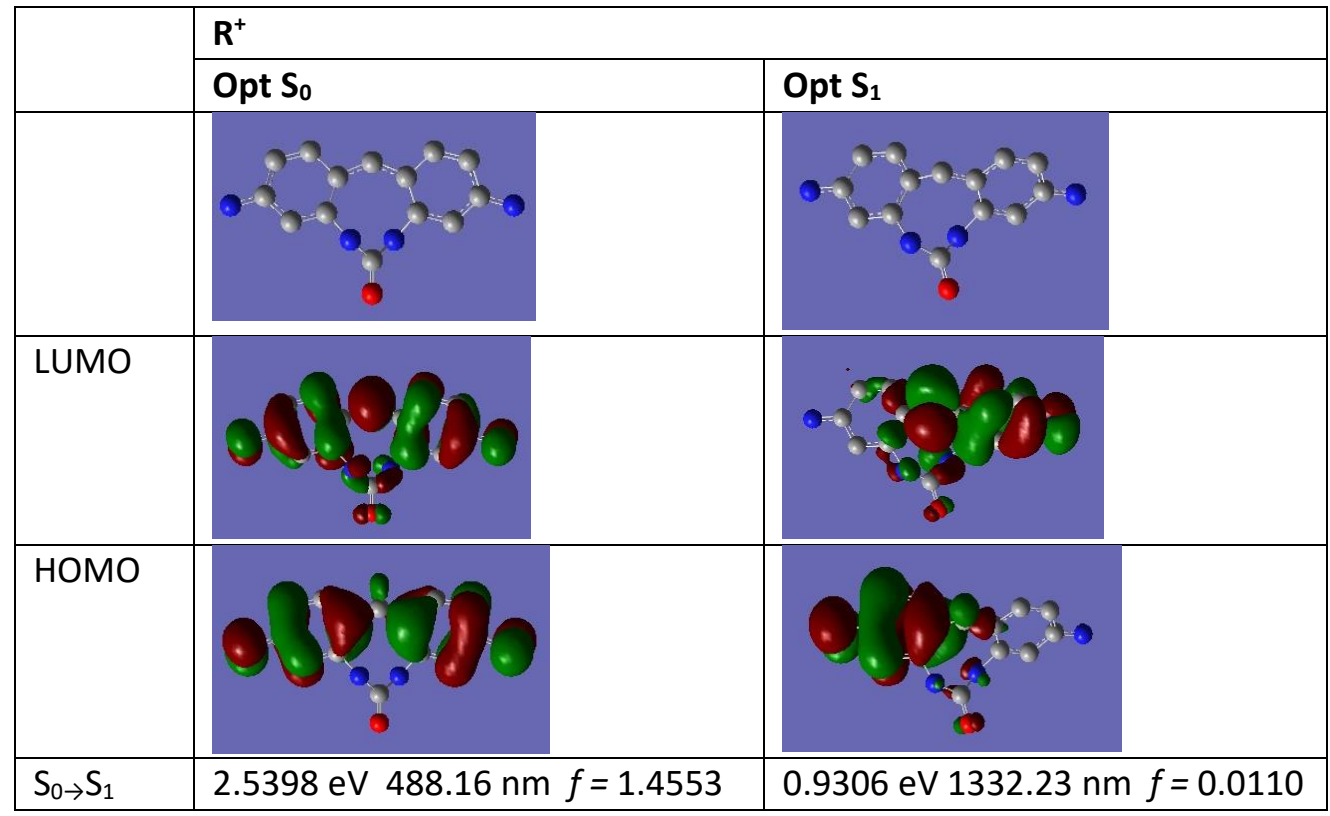

Table S7. xyz coordinates for $\mathrm{R}^{+}$optimized in $\mathrm{S}_{0}$ and $\mathrm{S}_{1}$ states. In bold - energies of optimized states.

\begin{tabular}{|c|c|c|c|c|c|c|c|}
\hline \multicolumn{4}{|c|}{$\begin{array}{l}S_{0} \\
-835.45567666 \\
-835.36233897\end{array}$} & \multicolumn{4}{|c|}{$\begin{array}{l}S_{1} \\
-835.409329007 \\
-835.375128232\end{array}$} \\
\hline & $x$ & $y$ & z & & $x$ & $y$ & $z$ \\
\hline C & -4.070 & -0.268 & -0.009 & $C$ & -3.870 & -0.579 & -0.436 \\
\hline C & 4.070 & -0.268 & 0.009 & C & 3.888 & -0.417 & -0.496 \\
\hline C & -3.665 & -1.570 & -0.422 & C & -3.113 & -1.784 & -0.617 \\
\hline C & 3.665 & -1.570 & 0.422 & C & 3.690 & -1.311 & 0.587 \\
\hline C & -3.072 & 0.659 & 0.382 & $C$ & -3.238 & 0.584 & 0.095 \\
\hline C & 3.072 & 0.659 & -0.383 & $C$ & 2.809 & 0.396 & -0.906 \\
\hline C & -2.338 & -1.891 & -0.421 & $C$ & -1.803 & -1.832 & -0.205 \\
\hline C & 2p.338 & -1.891 & 0.421 & $C$ & 2.448 & -1.430 & 1.164 \\
\hline C & -1.727 & 0.344 & 0.345 & $C$ & -1.928 & 0.503 & 0.505 \\
\hline C & 1.727 & 0.344 & -0.345 & $C$ & 1.558 & 0.285 & -0.317 \\
\hline C & -1.300 & -0.965 & -0.063 & C & -1.219 & -0.737 & 0.477 \\
\hline C & 1.300 & -0.965 & 0.063 & C & 1.312 & -0.705 & 0.691 \\
\hline
\end{tabular}




\begin{tabular}{|llll|llll|}
\hline $\mathrm{C}$ & 0.000 & 2.063 & 0.000 & $\mathrm{C}$ & -0.097 & 2.112 & 0.218 \\
$\mathrm{C}$ & 0.000 & -1.498 & 0.000 & $\mathrm{C}$ & 0.035 & -0.914 & 1.233 \\
$\mathrm{H}$ & -6.085 & -0.576 & -0.273 & $\mathrm{H}$ & -5.596 & -1.336 & -1.247 \\
$\mathrm{H}$ & 6.085 & -0.576 & 0.273 & $\mathrm{H}$ & 5.909 & -0.718 & -0.656 \\
$\mathrm{H}$ & -5.667 & 0.990 & 0.312 & $\mathrm{H}$ & -5.703 & 0.303 & -0.721 \\
$\mathrm{H}$ & 5.667 & 0.990 & -0.312 & $\mathrm{H}$ & 5.287 & 0.452 & -1.720 \\
$\mathrm{H}$ & -1.299 & 1.975 & 1.464 & $\mathrm{H}$ & -1.719 & 2.391 & 1.346 \\
$\mathrm{H}$ & 1.299 & 1.975 & -1.464 & $\mathrm{H}$ & 0.938 & 1.840 & -1.410 \\
$\mathrm{H}$ & 0.000 & -2.586 & 0.000 & $\mathrm{H}$ & -0.070 & -1.332 & 2.230 \\
$\mathrm{H}$ & -4.413 & -2.299 & -0.715 & $\mathrm{H}$ & -3.596 & -2.654 & -1.048 \\
$\mathrm{H}$ & 4.413 & -2.299 & 0.715 & $\mathrm{H}$ & 4.522 & -1.910 & 0.944 \\
$\mathrm{H}$ & -3.377 & 1.646 & 0.716 & $\mathrm{H}$ & -3.777 & 1.526 & 0.116 \\
$\mathrm{H}$ & 3.377 & 1.646 & -0.716 & $\mathrm{H}$ & 2.984 & 1.159 & -1.659 \\
$\mathrm{H}$ & -2.039 & -2.892 & -0.713 & $\mathrm{H}$ & -1.236 & -2.750 & -0.302 \\
$\mathrm{H}$ & 2.039 & -2.892 & 0.714 & $\mathrm{H}$ & 2.296 & -2.139 & 1.972 \\
$\mathrm{~N}$ & -5.369 & 0.074 & 0.014 & $\mathrm{~N}$ & -5.146 & -0.535 & -0.825 \\
$\mathrm{~N}$ & 5.369 & 0.074 & -0.015 & $\mathrm{~N}$ & 5.099 & -0.345 & -1.130 \\
$\mathrm{~N}$ & 0.827 & 1.322 & -0.848 & $\mathrm{~N}$ & 0.550 & 1.225 & -0.701 \\
$\mathrm{~N}$ & -0.827 & 1.321 & 0.848 & $\mathrm{~N}$ & -1.204 & 1.637 & 0.908 \\
$\mathrm{O}$ & 0.000 & 3.282 & 0.000 & $\mathrm{O}$ & 0.229 & 3.284 & 0.305 \\
\hline
\end{tabular}

Table S8. The forms of $\mathrm{R}^{+} \mathrm{Cl}^{-}$optimized in ground and excited states. Two forms, $A$ and $\mathrm{B}$, were found in the excited state. The coordinates for these forms are given in Table $\mathrm{S} 9$

\begin{tabular}{|c|c|c|c|}
\hline & \multicolumn{3}{|l|}{$\mathrm{R}^{+} \mathrm{Cl}^{-}$} \\
\hline & Opt So & Opt $S_{1} \quad$ B & Opt $S_{1} \quad A$ \\
\hline & 0 & & 0 \\
\hline LUMO & e & & \\
\hline HOMO & 8 & & \\
\hline & $2.5071 \mathrm{eV} 494.54 \mathrm{~nm} f=0.5913$ & $1.7569 \mathrm{eV} 705.71 \mathrm{~nm} f=0.0299$ & $0.9798 \mathrm{eV} 1265.36 \mathrm{~nm} f=0.0124$ \\
\hline
\end{tabular}


Table S9. xyz coordinates of $\mathrm{R}^{+} \mathrm{Cl}^{-}$forms of Mini Model optimized in $\mathrm{S}_{0}$ and $\mathrm{S}_{1}$ states. In bold - energies of optimized state.

\begin{tabular}{|c|c|c|c|c|c|c|c|c|c|c|c|}
\hline \multicolumn{4}{|c|}{$\begin{array}{l}S_{0} \\
-1295.83029772 \\
-1295.73816481\end{array}$} & \multicolumn{4}{|c|}{$\begin{array}{ll}S_{1} \quad B \\
-1295.8144173 \\
-1295.74985364\end{array}$} & \multicolumn{4}{|c|}{$\begin{array}{l}S_{1} \quad A \\
-1295.78913054 \\
-1295.75312099\end{array}$} \\
\hline & $x$ & $y$ & z & & $x$ & $y$ & z & & $x$ & $y$ & z \\
\hline C & 4.071 & -0.309 & -0.011 & C & -3.859 & -0.417 & 0.520 & C & 4.088 & -0.180 & -0.646 \\
\hline C & -4.071 & -0.309 & 0.013 & C & 4.290 & 0.263 & 0.104 & C & 3.605 & -1.094 & -0.410 \\
\hline C & 3.662 & 0.997 & -0.405 & C & -3.512 & 0.629 & 1.403 & C & -3.442 & -1.217 & -1.399 \\
\hline C & -3.662 & 0.997 & 0.406 & C & 3.792 & 1.571 & 0.288 & C & 3.221 & -2.374 & 0.064 \\
\hline C & 3.076 & -1.243 & 0.372 & C & -2.826 & -1.021 & -0.216 & C & -3.397 & 0.455 & 0.426 \\
\hline C & -3.077 & -1.243 & -0.370 & $C$ & 3.371 & -0.799 & 0.149 & C & 2.663 & -0.043 & -0.374 \\
\hline C & 2.334 & 1.316 & -0.399 & C & -2.202 & 1.036 & 1.508 & C & -2.188 & -1.643 & -1.031 \\
\hline C & -2.333 & 1.316 & 0.399 & C & 2.446 & 1.772 & 0.505 & C & 1.926 & -2.597 & 0.472 \\
\hline C & 1.730 & -0.930 & 0.340 & $C$ & -1.498 & -0.612 & -0.109 & C & -2.145 & 0.003 & 0.774 \\
\hline C & -1.730 & -0.930 & -0.340 & C & 2.010 & -0.593 & 0.367 & C & 1.361 & -0.262 & 0.053 \\
\hline C & 1.299 & 0.380 & -0.056 & C & -1.126 & 0.456 & 0.763 & C & -1.562 & -1.136 & 0.134 \\
\hline C & -1.299 & 0.380 & 0.055 & C & 1.484 & 0.717 & 0.552 & C & 0.923 & -1.583 & 0.409 \\
\hline C & 0.000 & -2.654 & -0.001 & C & 0.356 & -2.193 & -0.533 & C & -0.104 & 1.197 & 1.443 \\
\hline C & 0.000 & 0.917 & -0.001 & C & 0.147 & 1.083 & 0.887 & $C$ & -0.417 & -1.848 & 0.732 \\
\hline $\mathrm{H}$ & 6.086 & 0.006 & -0.276 & $H$ & -5.807 & -0.587 & 1.109 & $\mathrm{H}$ & -5.794 & -0.173 & -1.787 \\
\hline $\mathrm{H}$ & -6.085 & 0.005 & 0.280 & $\mathrm{H}$ & 6.190 & 0.806 & -0.406 & $H$ & 5.591 & -1.542 & -0.637 \\
\hline $\mathrm{H}$ & 5.671 & -1.568 & 0.289 & $\mathrm{H}$ & -5.331 & -1.722 & -0.037 & $\mathrm{H}$ & -5.785 & 0.966 & -0.491 \\
\hline $\mathrm{H}$ & -5.672 & -1.569 & -0.285 & $\mathrm{H}$ & 5.902 & -0.847 & -0.485 & $\mathrm{H}$ & 5.194 & 0.071 & -0.978 \\
\hline $\mathrm{H}$ & 1.314 & -2.572 & 1.448 & $\mathrm{H}$ & -1.046 & -1.666 & -1.785 & $\mathrm{H}$ & -1.821 & 1.140 & 2.487 \\
\hline $\mathrm{H}$ & -1.315 & -2.572 & -1.450 & $\mathrm{H}$ & 1.764 & -2.573 & 0.766 & $\mathrm{H}$ & 0.962 & 1.711 & -0.145 \\
\hline $\mathrm{H}$ & 0.000 & 2.010 & -0.001 & $\mathrm{H}$ & 0.086 & 2.069 & 1.341 & $\mathrm{H}$ & -0.667 & -2.694 & 1.366 \\
\hline $\mathrm{H}$ & 4.409 & 1.733 & -0.684 & $\mathrm{H}$ & -4.285 & 1.116 & 1.992 & $\mathrm{H}$ & -3.968 & -1.674 & -2.229 \\
\hline $\mathrm{H}$ & -4.409 & 1.733 & 0.685 & $\mathrm{H}$ & 4.474 & 2.416 & 0.264 & $\mathrm{H}$ & 3.950 & -3.178 & 0.083 \\
\hline $\mathrm{H}$ & 3.385 & -2.232 & 0.695 & $\mathrm{H}$ & -3.069 & -1.831 & -0.898 & $\mathrm{H}$ & -3.835 & 1.320 & 0.912 \\
\hline $\mathrm{H}$ & -3.386 & -2.232 & -0.693 & $\mathrm{H}$ & 3.733 & -1.815 & 0.016 & $\mathrm{H}$ & 2.969 & 0.966 & -0.634 \\
\hline $\mathrm{H}$ & 2.023 & 2.323 & -0.663 & $\mathrm{H}$ & -1.959 & 1.851 & 2.185 & $\mathrm{H}$ & -1.711 & -2.453 & -1.570 \\
\hline $\mathrm{H}$ & -2.023 & 2.323 & 0.662 & $\mathrm{H}$ & 2.085 & 2.786 & 0.654 & $\mathrm{H}$ & 1.629 & -3.588 & 0.801 \\
\hline$N$ & 5.372 & -0.649 & 0.004 & $\mathrm{~N}$ & -5.178 & -0.799 & 0.346 & $N$ & -5.311 & 0.229 & -0.995 \\
\hline$N$ & -5.372 & -0.649 & -0.001 & $\mathrm{~N}$ & 5.646 & 0.030 & -0.052 & $\mathrm{~N}$ & 4.872 & -0.882 & -0.894 \\
\hline $\mathrm{N}$ & -0.835 & -1.915 & -0.841 & $\mathrm{~N}$ & 1.204 & -1.774 & 0.488 & $\mathrm{~N}$ & 0.487 & 0.855 & 0.187 \\
\hline$N$ & 0.835 & -1.915 & 0.840 & $\mathrm{~N}$ & -0.567 & -1.255 & -0.990 & $\mathrm{~N}$ & -1.356 & 0.659 & 1.726 \\
\hline 0 & 0.001 & -3.874 & -0.002 & 0 & 0.418 & -3.324 & -1.005 & 0 & 0.387 & 2.020 & 2.198 \\
\hline $\mathrm{Cl}$ & 0.000 & 4.551 & -0.001 & $\mathrm{Cl}$ & -1.827 & 2.460 & -1.903 & $\mathrm{Cl}$ & 1.836 & 3.580 & -1.106 \\
\hline
\end{tabular}


Table S10. The torsion angles for central ring of ground and excited state forms of $\mathrm{R}^{+} \mathrm{Cl}^{-}$in Mini Model. It is seen that the central ring in form A has "broken symmetry" (the difference between the values of the 2345 and 5678 angles and 7812 and 8123 angles). The distances between $\mathrm{Cl}$ and $\mathrm{C} 5$ atoms in $\AA$ are at the bottom of the Table.

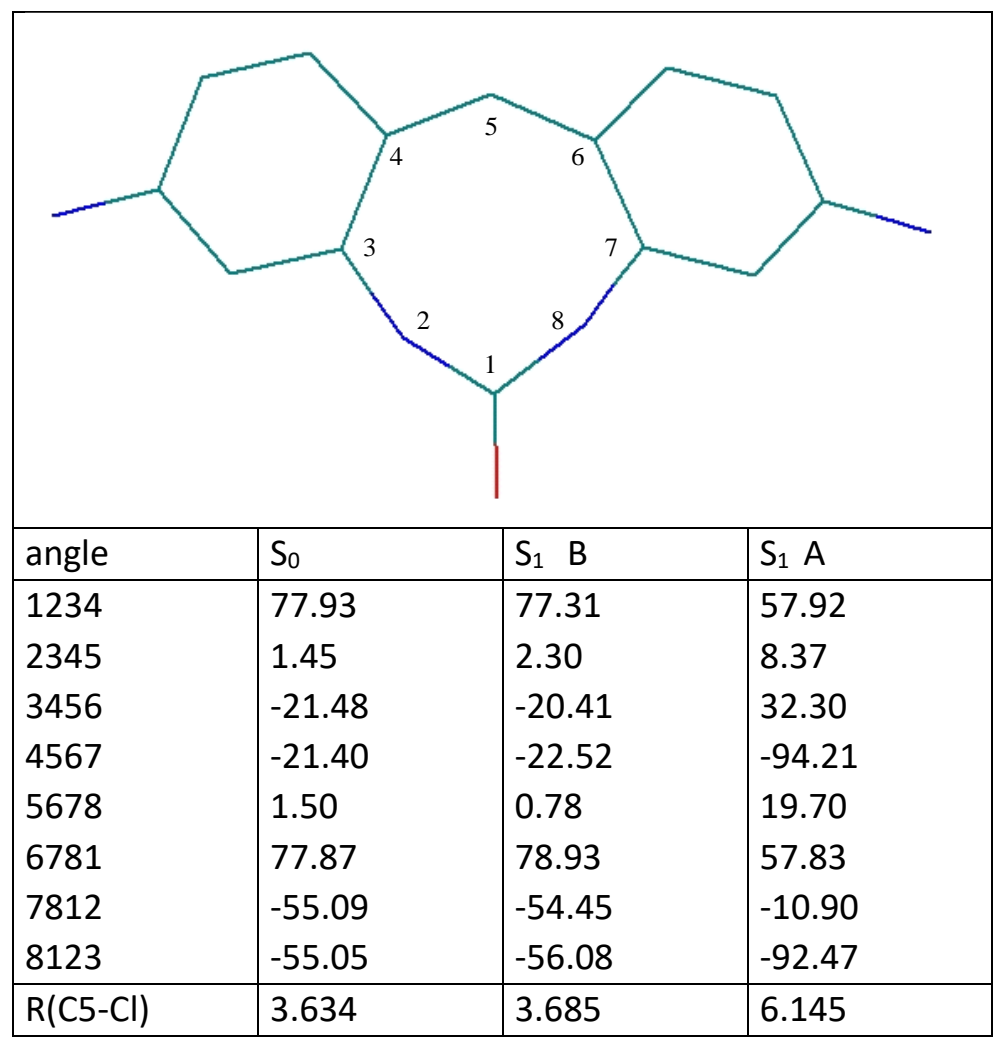

Table S11. The vibration frequencies corresponding to the optimized forms of $\mathrm{R}^{+}$and $\mathrm{R}^{+} \mathrm{Cl}^{-}$of Mini Model.

\begin{tabular}{|c|c|c|c|c|c|c|c|c|c|c|}
\hline & \multicolumn{4}{|l|}{$\mathrm{R}^{+}$} & \multicolumn{6}{|l|}{$\mathrm{R}^{+} \mathrm{Cl}^{-}$} \\
\hline & \multicolumn{2}{|l|}{$\mathrm{S}_{0}$} & \multicolumn{2}{|l|}{$S_{1}$} & \multicolumn{2}{|l|}{$\mathrm{S}_{0}$} & \multicolumn{2}{|l|}{ B $\quad S_{1}$} & \multicolumn{2}{|l|}{$A S_{1}$} \\
\hline & $\begin{array}{l}\mathrm{hv} \\
{\left[\mathrm{cm}^{-1}\right]}\end{array}$ & IR act. & $\begin{array}{l}\mathrm{hv} \\
{\left[\mathrm{cm}^{-1}\right]}\end{array}$ & IR act. & $\begin{array}{l}\text { hv } \\
{\left[\mathrm{cm}^{-1}\right]}\end{array}$ & IR act. & $\begin{array}{l}\mathrm{hv} \\
{\left[\mathrm{cm}^{-1}\right]}\end{array}$ & IR act. & $\begin{array}{l}\mathrm{hv} \\
{\left[\mathrm{cm}^{-1}\right]}\end{array}$ & IR act. \\
\hline 1 & 13.5 & 7.8 & 41.7 & 9.5 & 18.8 & 18.8 & 24.6 & 3.8 & 26.1 & 21.5 \\
\hline 2 & 63.8 & 8.0 & 50.8 & 7.3 & 37.5 & 9.8 & 32.9 & 1.0 & 31.0 & 12.9 \\
\hline 3 & 67.8 & 2.1 & 70.2 & 7.8 & 43.7 & 15.9 & 47.7 & 4.1 & 45.8 & 5.5 \\
\hline 4 & 78.2 & 1.3 & 93.2 & 0.5 & 64.5 & 10.3 & 66.3 & 10.7 & 59.5 & 28.9 \\
\hline 5 & 150.4 & 2.9 & 132.6 & 1.7 & 67.2 & 34.7 & 76.1 & 1.8 & 82.9 & 0.9 \\
\hline 6 & 185.3 & 0.5 & 189.4 & 6.1 & 80.0 & 4.1 & 85.2 & 8.3 & 95.7 & 2.3 \\
\hline 7 & 197.5 & 21.8 & 205.4 & 6.7 & 83.2 & 19.2 & 106.8 & 13.9 & 104.0 & 43.2 \\
\hline 8 & 244.8 & 4.5 & 240.7 & 3.7 & 153.5 & 2.2 & 150.7 & 1.5 & 139.4 & 1.8 \\
\hline 9 & 273.2 & 1.3 & 273.2 & 21.6 & 185.5 & 1.5 & 191.6 & 2.7 & 189.0 & 8.6 \\
\hline 10 & 287.4 & 11.9 & 297.6 & 8.8 & 199.8 & 22.5 & 217.8 & 4.6 & 205.0 & 9.5 \\
\hline 11 & 292.8 & 143.5 & 308.3 & 10.5 & 244.6 & 7.3 & 242.9 & 3.6 & 243.5 & 1.8 \\
\hline
\end{tabular}




\begin{tabular}{|c|c|c|c|c|c|c|c|c|c|c|}
\hline 12 & 292.9 & 463.4 & 338.4 & 18.2 & 263.3 & 62.7 & 278.2 & 3.7 & 277.9 & 22.9 \\
\hline 13 & 298.9 & 5.8 & 383.0 & 615.2 & 263.5 & 555.8 & 284.1 & 0.3 & 298.7 & 7.0 \\
\hline 4 & 335.4 & 3.8 & 390.0 & 285.5 & 273.3 & 4.9 & 326.3 & 15.1 & 311.1 & 8.4 \\
\hline 15 & 397.0 & 217.7 & 408.1 & 8.5 & 289.3 & 1.9 & 329.6 & 16.3 & 338.5 & 20.3 \\
\hline 16 & 421.3 & 12.3 & 425.2 & 47.4 & 298.4 & 1.2 & 351.7 & 25.7 & 385.6 & 39.1 \\
\hline 17 & 434.6 & 16.6 & 434.1 & 10.6 & 334.8 & 2.5 & 356.3 & 31.8 & 405.5 & 524.3 \\
\hline 18 & 460.0 & 13.3 & 438.1 & 2.3 & 399.7 & 187.6 & 388.9 & 60.9 & 411.8 & 109.9 \\
\hline 19 & 466.1 & 0.7 & 453.7 & 21.4 & 421.4 & 16.5 & 413.6 & 7.9 & 417.6 & 335.2 \\
\hline 20 & 481.6 & 2.5 & 490.3 & 10.4 & 438.9 & 30.1 & 425.8 & 1.1 & 434.1 & 7.0 \\
\hline 21 & 500.9 & 292.5 & 514.0 & 14.1 & 465.1 & 12.6 & 461.9 & 78.2 & 440.1 & 37.1 \\
\hline 22 & 525.0 & 0.0 & 535.6 & 110.6 & 469.1 & 1.0 & 467.0 & 288.5 & 461.2 & 28.0 \\
\hline 23 & 525.3 & 1.9 & 548.6 & 204.4 & 480.5 & 2.2 & 479.4 & 1.2 & 494.0 & 16.5 \\
\hline 24 & 533.4 & 4.5 & 557.8 & 3.9 & 502.0 & 267.6 & 487.7 & 1431.1 & 514.4 & 9.9 \\
\hline 25 & 591.7 & 0.0 & 573.4 & 4.3 & 520.3 & 0.1 & 521.4 & 19.8 & 527.4 & 245.2 \\
\hline 26 & 612.1 & 54.4 & 596.7 & 91.4 & 520.6 & 2.1 & 527.4 & 535.5 & 544.4 & 72.8 \\
\hline 27 & 616.3 & 0.0 & 603.5 & 24.8 & 535.4 & 5.0 & 530.1 & 233.2 & 558.9 & 6.1 \\
\hline 28 & 647.2 & 48.5 & 635.4 & 87.8 & 592.4 & 0.3 & 591.6 & 5.4 & 577.2 & 39.7 \\
\hline 29 & 674.6 & 3.6 & 638.6 & 24.4 & 612.5 & 53.8 & 614.6 & 0.9 & 595.4 & 82.0 \\
\hline 30 & 712.6 & 61.8 & 676.1 & 92.9 & 616.4 & 0.2 & 616.4 & 18.2 & 608.8 & 22.0 \\
\hline 31 & 723.9 & 0.0 & 703.5 & 15.0 & 648.7 & 40.5 & 624.8 & 126.1 & 632.2 & 86.4 \\
\hline 32 & 737.7 & 98.0 & 714.2 & 16.6 & 674.8 & 6.4 & 662.3 & 10.5 & 639.0 & 32.7 \\
\hline 33 & 743.2 & 11.2 & 723.4 & 6.9 & 714.3 & 45.0 & 698.8 & 12.1 & 675.2 & 67.8 \\
\hline 34 & 793.2 & 0.0 & 785.6 & 299.5 & 724.7 & 0.1 & 719.0 & 4.9 & 702.6 & 9.9 \\
\hline 35 & 814.8 & 62.3 & 790.2 & 59.0 & 738.2 & 105.7 & 732.8 & 45.2 & 714.9 & 13.7 \\
\hline 36 & 830.6 & 83.2 & 800.3 & 72.6 & 741.7 & 5.2 & 736.8 & 11.4 & 727.2 & 5.7 \\
\hline 37 & 832.4 & 5.7 & 818.0 & 34.9 & 792.7 & 0.8 & 774.3 & 26.7 & 784.6 & 392.0 \\
\hline 38 & 847.5 & 245.5 & 843.2 & 38.2 & 818.6 & 86.0 & 787.4 & 1.0 & 796.3 & 49.9 \\
\hline 39 & 860.8 & 19.8 & 848.8 & 49.7 & 832.5 & 47.2 & 825.2 & 75.8 & 807.1 & 29.3 \\
\hline 40 & 869.4 & 123.2 & 860.0 & 15.6 & 835.1 & 7.2 & 829.2 & 6.1 & 819.8 & 58.1 \\
\hline 41 & 887.7 & 4.8 & 870.6 & 28.7 & 846.0 & 240.9 & 834.5 & 8.8 & 849.9 & 58.0 \\
\hline 42 & 892.9 & 50.2 & 878.6 & 58.3 & 858.6 & 18.7 & 842.9 & 236.4 & 859.6 & 22.0 \\
\hline 43 & 936.3 & 460.8 & 921.0 & 32.8 & 867.2 & 114.9 & 855.8 & 10.6 & 865.6 & 17.8 \\
\hline 44 & 937.1 & 4.2 & 955.4 & 22.6 & 886.9 & 5.7 & 862.3 & 59.2 & 876.2 & 29.6 \\
\hline 45 & 979.1 & 0.1 & 968.3 & 379.7 & 893.4 & 55.4 & 884.4 & 4.4 & 893.7 & 81.4 \\
\hline 46 & 982.1 & 146.5 & 986.8 & 147.8 & 936.2 & 7.3 & 891.8 & 23.1 & 942.2 & 96.2 \\
\hline 47 & 987.5 & 14.1 & 1008.0 & 95.2 & 971.2 & 262.7 & 936.6 & 6.8 & 951.3 & 192.8 \\
\hline 48 & 1017.8 & 12.6 & 1036.9 & 5220.1 & 988.6 & 21.8 & 953.9 & 2.4 & 962.5 & 1482.8 \\
\hline 49 & 1070.8 & 27.6 & 1070.4 & 240.9 & 992.1 & 3.4 & 954.5 & 2.4 & 983.9 & 358.7 \\
\hline 50 & 1082.2 & 2.3 & 1094.5 & 10.3 & 1008.3 & 402.7 & 991.4 & 1.1 & 1002.0 & 2043.1 \\
\hline 51 & 1152.8 & 84.7 & 1156.4 & 97.7 & 1019.9 & 10.0 & 1029.1 & 9.1 & 1006.7 & 1266.0 \\
\hline 52 & 1182.9 & 50.1 & 1168.9 & 185.2 & 1070.5 & 31.5 & 1101.8 & 10.7 & 1065.5 & 18.6 \\
\hline 53 & 1216.1 & 955.1 & 1217.5 & 684.6 & 1081.7 & 3.6 & 1111.0 & 0.6 & 1092.2 & 6.0 \\
\hline 54 & 1249.8 & 53.5 & 1226.0 & 151.4 & 1152.4 & 119.6 & 1150.2 & 100.5 & 1157.2 & 112.0 \\
\hline 55 & 1256.2 & 676.7 & 1237.4 & 114.2 & 1181.4 & 32.1 & 1172.1 & 48.6 & 1165.7 & 57.9 \\
\hline
\end{tabular}




\begin{tabular}{|c|c|c|c|c|c|c|c|c|c|c|}
\hline 56 & 1277.5 & 3.6 & 1246.3 & 27.7 & 1221.8 & 763.4 & 1210.9 & 3.4 & 1215.4 & 691.6 \\
\hline 57 & 1300.3 & 116.7 & 1267.7 & 199.2 & 1250.9 & 71.3 & 1226.3 & 35.5 & 1224.7 & 155.7 \\
\hline 58 & 1304.9 & 2.4 & 1277.2 & 599.1 & 1257.2 & 769.8 & 1237.2 & 40.6 & 1238.6 & 114.2 \\
\hline 59 & 1366.8 & 40.8 & 1314.9 & 1740.5 & 1276.7 & 0.0 & 1239.6 & 281.2 & 1245.9 & 28.7 \\
\hline 60 & 1389.7 & 553.0 & 1351.3 & 4.0 & 1305.6 & 3.9 & 1289.6 & 192.3 & 1267.5 & 147.9 \\
\hline 61 & 1390.5 & 8.9 & 1366.7 & 1.2 & 1308.4 & 212.9 & 1290.4 & 23.3 & 1273.7 & 753.8 \\
\hline 62 & 1437.1 & 3293.8 & 1385.5 & 101.5 & 1373.1 & 64.7 & 1341.7 & 285.9 & 1303.2 & 977.9 \\
\hline 63 & 1440.8 & 84.6 & 1411.8 & 180.6 & 1389.7 & 3.3 & 1344.2 & 51.5 & 1351.5 & 21.1 \\
\hline 64 & 1455.7 & 29.3 & 1438.8 & 29.7 & 1391.0 & 496.6 & 1372.8 & 189.7 & 1367.8 & 3.4 \\
\hline 65 & 1459.5 & 1.3 & 1452.8 & 226.8 & 1437.5 & 79.8 & 1379.2 & 241.8 & 1381.2 & 89.3 \\
\hline 66 & 1510.3 & 909.4 & 1496.0 & 257.7 & 1438.1 & 3169.0 & 1395.4 & 387.9 & 1411.2 & 158.7 \\
\hline 67 & 1535.1 & 210.5 & 1503.2 & 150.8 & 1458.6 & 0.5 & 1436.0 & 4.9 & 1433.2 & 81.6 \\
\hline 68 & 1552.3 & 756.3 & 1519.1 & 213.8 & 1459.9 & 414.8 & 1441.9 & 3.1 & 1457.8 & 257.9 \\
\hline 69 & 1562.6 & 38.0 & 1538.8 & 956.0 & 1514.0 & 399.8 & 1473.4 & 104.5 & 1497.1 & 93.8 \\
\hline 70 & 1568.8 & 131.9 & 1556.2 & 48.5 & 1532.4 & 218.8 & 1505.4 & 22.4 & 1505.0 & 90.2 \\
\hline 71 & 1571.0 & 143.9 & 1559.1 & 1983.6 & 1555.8 & 597.2 & 1527.8 & 106.6 & 1521.5 & 245.5 \\
\hline 72 & 1595.4 & 3816.5 & 1578.9 & 362.6 & 1563.5 & 41.7 & 1534.6 & 206.5 & 1537.7 & 1363.2 \\
\hline 73 & 1654.7 & 1181.9 & 1650.4 & 269.0 & 1568.6 & 157.0 & 1546.6 & 19.7 & 1556.4 & 1475.9 \\
\hline 74 & 1667.9 & 14.1 & 1658.6 & 595.6 & 1574.7 & 313.2 & 1581.5 & 133.1 & 1560.5 & 165.5 \\
\hline 75 & 1671.2 & 852.5 & 1673.6 & 248.5 & 1603.3 & 3868.6 & 1591.3 & 34.1 & 1586.7 & 111.9 \\
\hline 76 & 1683.6 & 40.3 & 1688.7 & 249.9 & 1653.8 & 974.1 & 1641.6 & 830.1 & 1649.3 & 112.8 \\
\hline 77 & 1792.3 & 783.9 & 1776.0 & 964.5 & 1666.5 & 14.2 & 1652.7 & 5.1 & 1656.4 & 553.2 \\
\hline 78 & 3163.7 & 6.0 & 3181.9 & 0.5 & 1670.2 & 784.0 & 1661.3 & 795.9 & 1669.2 & 168.3 \\
\hline 79 & 3199.3 & 16.7 & 3184.0 & 14.4 & 1681.6 & 43.7 & 1665.5 & 53.6 & 1682.4 & 47.5 \\
\hline 80 & 3199.4 & 8.7 & 3194.1 & 7.3 & 1788.7 & 793.3 & 1767.5 & 845.5 & 1771.4 & 941.1 \\
\hline 81 & 3201.0 & 0.0 & 3209.2 & 16.8 & 3088.0 & 162.3 & 3162.2 & 17.4 & 3178.4 & 1.6 \\
\hline 82 & 3201.4 & 3.0 & 3223.7 & 1.7 & 3194.4 & 0.2 & 3179.6 & 36.6 & 3179.8 & 6.5 \\
\hline 83 & 3217.2 & 38.4 & 3224.3 & 2.8 & 3194.8 & 8.0 & 3181.1 & 37.5 & 3190.5 & 11.5 \\
\hline 84 & 3217.6 & 7.2 & 3236.6 & 0.6 & 3197.5 & 16.5 & 3183.1 & 22.3 & 3205.9 & 26.2 \\
\hline 85 & 3567.0 & 69.8 & 3553.2 & 71.6 & 3197.7 & 9.5 & 3183.7 & 4.5 & 3220.3 & 4.7 \\
\hline 86 & 3568.0 & 40.6 & 3584.1 & 210.6 & 3212.0 & 53.7 & 3199.1 & 54.0 & 3220.8 & 1.9 \\
\hline 87 & 3603.7 & 1495.7 & 3596.3 & 92.1 & 3212.3 & 0.3 & 3199.8 & 36.5 & 3233.5 & 150.0 \\
\hline 88 & 3606.2 & 24.4 & 3601.1 & 120.6 & 3566.9 & 66.9 & 3569.2 & 46.4 & 3234.6 & 1062.2 \\
\hline 89 & 3730.2 & 91.4 & 3706.4 & 133.9 & 3567.9 & 39.4 & 3570.1 & 24.0 & 3584.6 & 248.9 \\
\hline 90 & 3730.2 & 96.0 & 3711.9 & 52.6 & 3605.5 & 1400.6 & 3574.0 & 129.9 & 3590.1 & 90.5 \\
\hline 91 & & & & & 3607.9 & 24.5 & 3575.3 & 127.8 & 3606.0 & 122.6 \\
\hline 92 & & & & & 3732.2 & 89.7 & 3681.6 & 33.0 & 3704.8 & 47.6 \\
\hline 93 & & & & & 3732.2 & 91.4 & 3683.8 & 35.3 & 3707.8 & 131.4 \\
\hline
\end{tabular}




\section{Photoluminescence data}

Table S12. The photoluminescence quantum yields between polycrystalline state and the ground powder.

\begin{tabular}{|c|c|c|}
\hline \multirow{2}{*}{ Compd. } & \multicolumn{2}{|c|}{$\Phi / \%$} \\
\cline { 2 - 3 } & Original polycrystalline state & The ground powder \\
\hline FA18 & 0.31 & 0.28 \\
FA23 & 0.11 & 0.11 \\
FA34 & 0.13 & 0.09 \\
FA36 & 0.09 & 0.06 \\
\hline
\end{tabular}

\section{References}

(1) Frisch, M. J.; Trucks, G. W.; Schlegel, H. B.; Scuseria, G. E.; Robb, M. A.; Cheeseman, J. R.; Scalmani, G.; Barone, V.; Mennucci, B.; Petersson, G. A.; et al. Gaussian 09, Revision D.01. Gaussian, Inc.: Wallingford CT 2013. 